\title{
Verification of an ADER-DG method for complex dynamic rupture problems
}

\author{
C. Pelties ${ }^{1}$, A.-A. Gabriel $^{1}$, and J.-P. Ampuero ${ }^{2}$ \\ ${ }^{1}$ Department of Earth and Environmental Sciences, Geophysics, Ludwig-Maximilians-Universität, München, Germany \\ ${ }^{2}$ Seismological Laboratory, California Institute of Technology, Pasadena, California, USA \\ Correspondence to: C. Pelties (pelties@lmu.de)
}

Received: 8 November 2013 - Published in Geosci. Model Dev. Discuss.: 28 November 2013

Revised: 14 March 2014 - Accepted: 18 March 2014 - Published: 13 May 2014

\begin{abstract}
We present results of thorough benchmarking of an arbitrary high-order derivative discontinuous Galerkin (ADER-DG) method on unstructured meshes for advanced earthquake dynamic rupture problems. We verify the method by comparison to well-established numerical methods in a series of verification exercises, including dipping and branching fault geometries, heterogeneous initial conditions, bimaterial interfaces and several rate-and-state friction laws. We show that the combination of meshing flexibility and high-order accuracy of the ADER-DG method makes it a competitive tool to study earthquake dynamics in geometrically complicated setups.
\end{abstract}

\section{Introduction}

The combined simulation of dynamic fault rupture and seismic wave propagation is a useful tool to gain insight into the poorly constrained processes of earthquake faulting. Dynamic rupture modeling aims to understand the underlying physics governing earthquakes and may be incorporated in physics-based seismic hazard assessment and strong motion prediction in preparation of future, possibly devastating, events (Ely et al., 2010; Harris et al., 2011; Roten et al., 2011).

Recent advances in dynamic rupture simulations furthered our understanding of the earthquake cycle in the Parkfield region (Barbot et al., 2012), the influence of low velocity fault zones (Huang and Ampuero, 2011) and off-fault plasticity (Templeton and Rice, 2008; Ma and Andrews, 2010; Gabriel et al., 2013) on the dynamics of the fracture process, mechanisms to generate pulse-like and supershear earthquakes and their consequences (Dunham, 2007;
Daub et al., 2010; Gabriel et al., 2012) and the interaction between fault branches (Oglesby et al., 2003; Bhat et al., 2007; DeDontney et al., 2011). Dynamically consistent predictions of strong ground-motion excitation in earthquake scenarios have been pushing computational performance to the petaflop scale (Cui et al., 2010; Zhou et al., 2013).

Nevertheless, despite the current achievements, the numerical simulation of the rupture process and its implementation into elastodynamics solvers poses challenges. For instance, the discontinuity of displacements across the fault has to be represented accurately and accounted for by the computational mesh. Furthermore, the dynamic rupture implementation in many numerical methods, such as finite difference, finite element and spectral element methods, suffers from spurious high-frequency oscillations, which may affect the nonlinear interaction between waves and dynamic rupture and potentially contaminate the solution over all space-time scales (Duan and Day, 2008). Thus, numerical regularization, artificial attenuation or smoothing is usually necessary to suppress high-frequency numerical noise with spatial scale near the resolution limit of the mesh. Such artificial damping mechanisms, as for example the deployment of a thin layer of Kelvin-Voigt viscous material surrounding the fault (e.g., Ampuero, 2008; Day et al., 2005; Dalguer and Day, 2007) are not completely satisfying. The physical solution is not necessarily insensitive to the precise parametrization of the added damping. The damping may interfere with the actual physics of interest, for example by slowing down the rupture propagation (Andrews, 2005) and smoothing out small-scale features. The artificial damping may also reduce the timestep length and thus increase the computational effort considerably. 
Realistic earthquake scenario simulations would ideally cover a frequency range relevant for engineering applications (up to $20 \mathrm{~Hz}$ ), and include geological models spanning hundreds of kilometers consisting of complicated fault geometries, topography, oceans and low velocity sedimentary basins. Simultaneously, fine spatial resolution is required to resolve the frictional weakening in the cohesive zone at the rupture tip with a sufficient number of computational nodes or elements, at scales down to tens of meters or less (Day et al., 2005). The constitutive laws describing the frictional sliding of faults originate in laboratory experiments carried out on much smaller scales than in natural setups (Dieterich, 1978; Ohnaka and Kuwahara, 1990; Di Toro et al., 2005). While it is still uncertain how to extrapolate fault constitutive properties from laboratory to natural scales, testing the large-scale implications of laboratory friction poses an enormous computational challenge. Additionally, the propagation of small wavelengths over large distances accumulates numerical dispersion and diffusion errors and motivates the adoption of a high-order accurate discretization. Another challenge for dynamic rupture simulations are large uncertainties in initial conditions and fault constitutive parameters. The rupture evolution depends strongly on model parameters such as initial background stresses, nucleation procedure and frictional properties. Forward modeling of dynamic rupture can support the search for proper friction models and model parameters (Cochard and Madariaga, 1994; Day et al., 1998; Aochi et al., 2003; Kaneko et al., 2008; Brietzke et al., 2009).

In order to avoid additional errors that interfere with the physical problem, accurate numerical methods that produce reliable results are desirable. Furthermore, computational efficiency, parallelization and high scalability are crucial demands for numerical methods simulating realistic earthquake scenarios.

Here, we present a thorough verification of the software SeisSol (Käser and Dumbser, 2006; Dumbser and Käser, 2006), a high-order derivative discontinuous Galerkin (ADER-DG) method on unstructured meshes, for advanced dynamic rupture problems (de la Puente et al., 2009; Pelties et al., 2012). In contrast to the well verified and validated simulation of seismic wave propagation, the verification process of spontaneous dynamic rupture simulations suffers from the lack of analytical reference solutions. Therefore, we verify the performance of the ADER-DG method in the benchmark suite established by the Southern California Earthquake Center (SCEC) (Harris et al., 2009, 2011). All simulation results presented here are available at http://scecdata.usc.edu/cvws/. The test problems cover many important aspects in realistic faulting setups, including dipping and branching fault geometries, bimaterial interfaces, heterogeneous initial conditions and different formulations of rate-and-state friction laws.

Our results demonstrate the benefits of SeisSol for dynamic rupture and ground motion simulations in realistic settings. Importantly, we confirm the lack of systematic numerical artifacts in advanced faulting setups, as reported for the basic example of a planar fault embedded in a homogeneous full space (SCEC test case TPV3) by Pelties et al. (2012).

\section{Numerical method}

De la Puente et al. (2009) and Pelties et al. (2012) presented a new, alternative numerical scheme for the simulation of earthquake faulting. The underlying solver for wave propagation is an ADER-DG method (Käser and Dumbser, 2006; Dumbser and Käser, 2006) with high order accuracy in space and time, based on tetrahedral element discretization. Between any two elements information is exchanged via numerical fluxes. We briefly outline the algorithm to evaluate the friction law in Appendix A and extend this approach to faulting at dissimilar material contacts. For further details on the concept, the reader is referred to de la Puente et al. (2009) and Pelties et al. (2012). The software package SeisSol provides pre- and postprocessing tools including interfaces to external mesh generators and a mesh partitioning concept based on METIS (Karypis and Kumar, 1998).

The use of a tetrahedral-element discretization leads to rapid and automatized mesh generation that can be readily aligned to complex geometrical features. The possibility of mesh refinement is advantageous for dynamic rupture problems as the mesh resolution can be adapted to areas of interest, such as the fault plane or complex topography. Furthermore, the mesh size can be coarsened with increasing distance to the fault to reduce the computational cost. No artificial reflections due to mesh coarsening are observed (de la Puente et al., 2009).

In contrast to the typically applied traction at split-node approach (Andrews, 1999; Day et al., 2005; Dalguer and Day, 2007), ADER-DG solves the frictional sliding via the inverse Riemann problem (Toro, 1999; LeVeque, 2002), in which the exact solution is modified to incorporate frictional boundary conditions. Solving the inverse Riemann problem inherits the favorable numerical properties from the exact Riemann solver or Godunov flux. The numerical properties of the ADER-DG algorithm are extremely sensitive to the choice of flux function. In particular, our implementation introduces a very selective numerical dissipation by employing an upwind flux, as discussed in Pelties et al. (2012). The dissipation increases with increasing frequencies and is stronger beyond a cut-off frequency that depends on the mesh size $h$ and on the order of accuracy $\mathcal{O}$. The cutoff frequency is expected to be inversely proportional to the travel time of $\mathrm{S}$ waves over a typical grid spacing $\sim h / \mathcal{O} / c_{\mathrm{S}}$. Higher frequency modes are subdued while the physically meaningful lower frequencies are minimally affected. This is advantageous for dynamic rupture simulations: spurious highfrequency oscillations are not generated in the first place and, thus, no additional damping procedures need to be applied. 

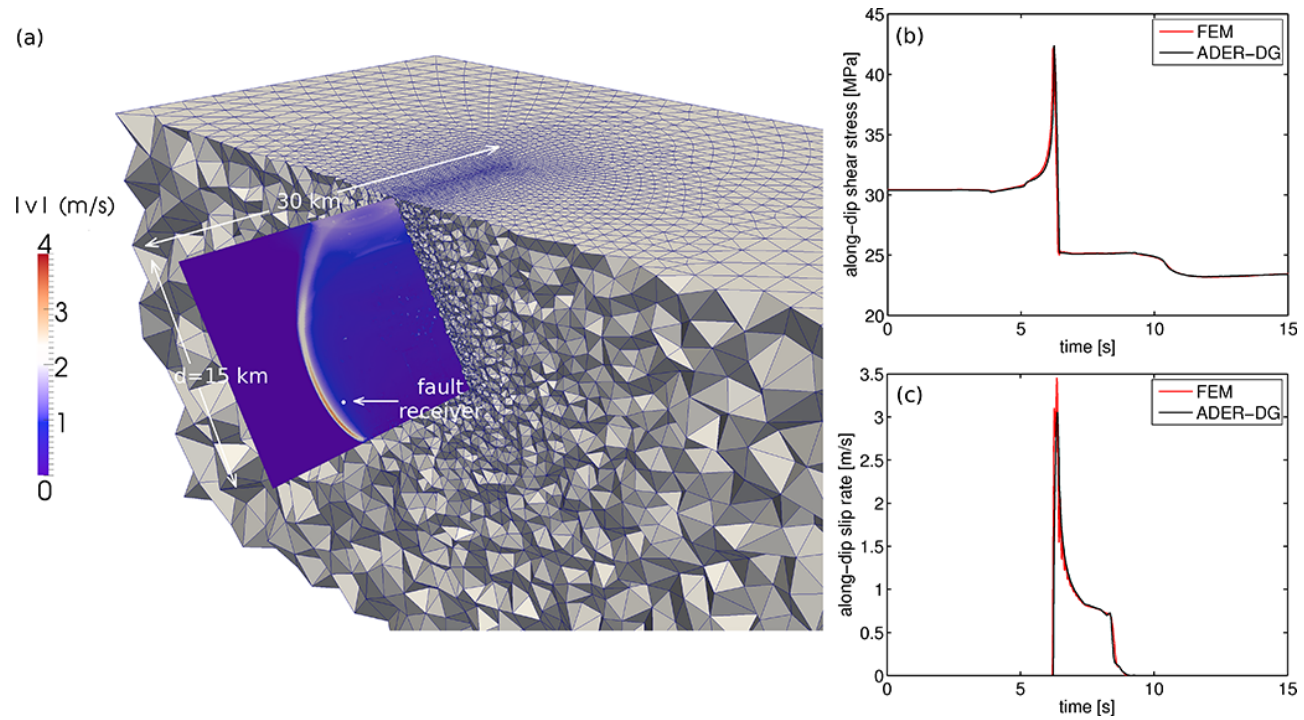

Fig. 1. (a) Asymmetric, unstructured mesh discretizing the dipping fault setup of benchmark problems TPV10 and TPV11 for SeisSol, and absolute slip rate $|v|$ on the fault plane at $t=7.4 \mathrm{~s}$ of TPV10 (subshear scenario). (b) Along-dip slip rate and (c) along-dip shear stress on a fault location at $7.5 \mathrm{~km}$ along-strike and $12 \mathrm{~km}$ along-dip in TPV10. The ADER-DG solution is shown in black, the FEM solution in red.

We compare our results to those of the well-established software FaultMod (Barall, 2009), a finite element (FE) code implemented on hexahedral elements and designed specifically for constructing physics-based models of fault systems that involve complex 3-D geometry and 3-D variation of material properties. The implementation of fault friction is based on the traction-at-split-nodes method. Faults are represented using common and differential nodes, which have a nondiagonal mass matrix. Also, FaultMod uses an implicit time stepping scheme, where displacement, velocities, and acceleration are computed simultaneously. The method implements Newmark damping (Hughes, 2000) and an optional thin viscous layer surrounding the fault zone (Day et al., 2005; Dalguer and Day, 2007) to suppress spurious highfrequency oscillations. For specific details about damping tuning strategies we refer to Rojas et al. (2008) and Barall (2009). In all benchmarks presented here, FaultMod applies a grid-doubling technique to enable high resolution at the fault and its immediate surroundings, and coarser grid spacing away from the fault.

In Sect. 7 we additionally show a comparison of our results with other high-order dynamic rupture codes based on a variety of numerical methods.

\section{Dip-slip fault with depth-dependent background stress}

Many studies of earthquake source physics have focused on vertical strike-slip faulting. Nevertheless, many faults exhibit dipping planes, including subduction zone faults in which most of the seismic energy was released over the last century (Pacheco and Sykes, 1992). The asymmetric geometry of dipping faults is of particular interest since it results in asymmetric near-source ground motion (Oglesby et al., 1998). Furthermore, if the rupture front reaches the surface it strongly excites seismic waves (Madariaga, 2003) and interacts with reflected waves from the free surface (Huang et al., 2013). Reproducing these effects accurately in numerical simulations poses challenges in terms of mesh generation and numerical stability.

We model spontaneous rupture on a $60^{\circ}$ dipping normal fault reaching the surface of a homogeneous half-space. Subshear (SCEC test case TPV10) and supershear (SCEC test case TPV11) conditions are set by varying the value of the static friction coefficient. Rupture is initiated by setting the along-dip component of background shear stress slightly above the static yield strength in a pre-defined nucleation patch. In order to accurately sample the initial stress and material parameters, we assign individual values to each Gaussian integration point (GP) used in the friction solver (Pelties et al., 2012). Slip-weakening friction on the fault and linear elasticity in the bulk are assumed. All simulation parameters are summarized in Table 1.

We compare the ADER-DG $\mathcal{O} 5$ solution on elements with edge length of $200 \mathrm{~m}$ to the finite element method solution computed by the FaultMod code of Barall (2009) with an edge length of $100 \mathrm{~m}$ and $\mathcal{O} 2$. Our mesh was gradually coarsened up to maximum edge lengths of $5 \mathrm{~km}$ away from the fault in order to concentrate numerical cost where needed. Figure 1a depicts our asymmetric unstructured mesh and a snapshot of the absolute slip velocity on the dipping fault plane from our simulation. 

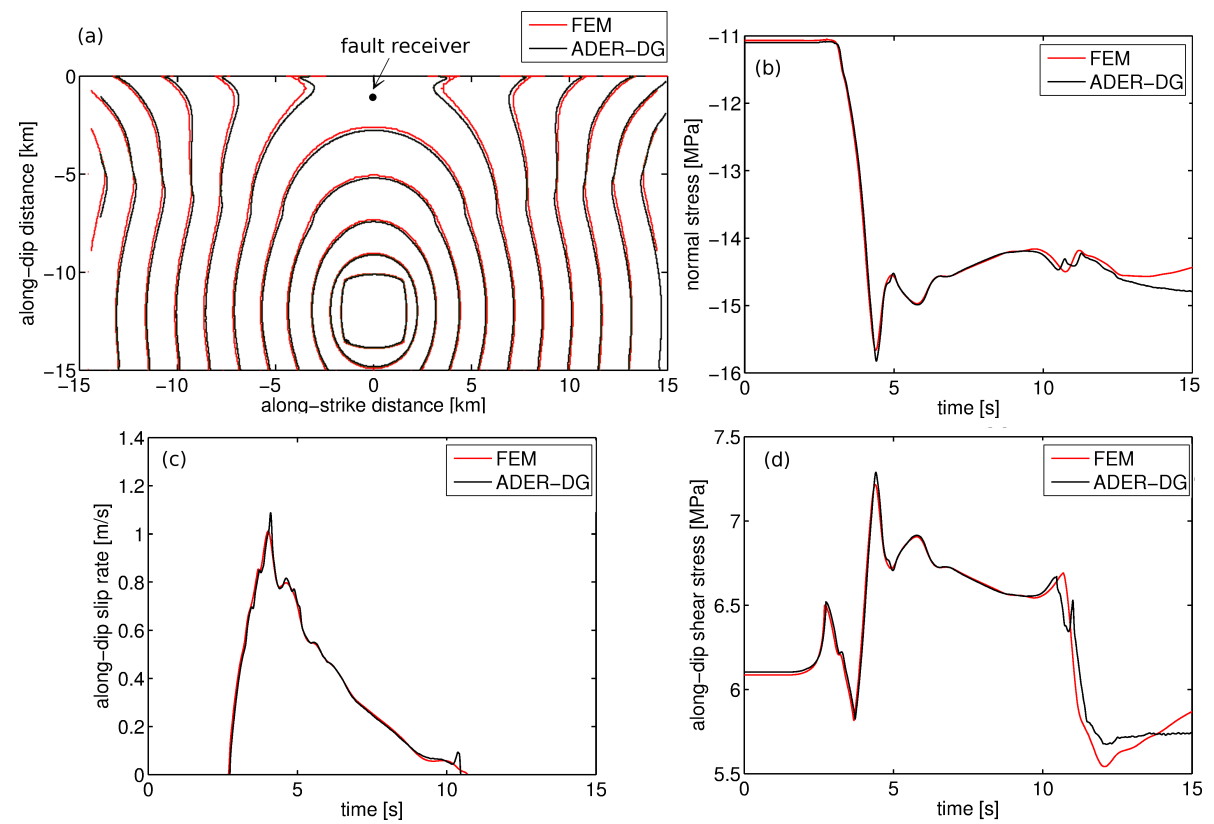

Fig. 2. Supershear and free surface effects in TPV11. (a) Rupture front contours on the fault plane every 0.5 s. (b) Normal stress, (c) along-dip slip rate and (d) along-dip shear stress at an on-fault receiver located $0 \mathrm{~km}$ along-strike and $1.5 \mathrm{~km}$ along-dip. The ADER-DG solution is shown in black, the FEM comparison solution in red.

Table 1. Simulation parameters for SCEC test cases TPV10 and TPV11.

\begin{tabular}{llr}
\hline$c_{\mathrm{P}}$ & P wave speed & $5716 \mathrm{~m} \mathrm{~s}^{-1}$ \\
$c_{\mathrm{S}}$ & Shear wave speed & $3300 \mathrm{~m} \mathrm{~s}^{-1}$ \\
$\rho$ & Density & $2700 \mathrm{~kg} \mathrm{~m}^{-3}$ \\
$\mu_{\mathrm{S}}$ & Static friction coefficient TPV10 & 0.76 \\
$\mu_{\mathrm{S}}$ & Static friction coefficient TPV11 & 0.57 \\
$\mu_{\mathrm{d}}$ & Dynamic friction coefficient & 0.448 \\
$c$ & Cohesion & $0.2 \mathrm{MPa}$ \\
$D_{\mathrm{c}}$ & Slip-weakening critical distance & $0.5 \mathrm{~m}$ \\
$d$ & Down-dip distance & $0 \ldots 15 \mathrm{~km}$ \\
$\sigma_{0}$ & Background normal stress & $d \times 7378 \mathrm{~Pa} \mathrm{~m}^{-1}$ \\
$\tau_{0}$ & Background shear stress along-dip & $0.55 \sigma_{0}$ \\
$\tau_{\text {nuc }}$ & Nucleation shear stress along-dip & $\left(\mu_{\mathrm{S}}+0.0057\right) \sigma_{0}+c$ \\
$A_{\text {nuc }}$ & Nucleation size & $3 \mathrm{~km} \times 3 \mathrm{~km}$ \\
$A_{\text {fault }}$ & Faulting area & $30 \mathrm{~km} \times 15 \mathrm{~km}$ \\
$h$ & Element edge length & $200 \mathrm{~m}$ \\
$\mathcal{O}$ & Spatiotemporal order of accuracy & 5 \\
\hline
\end{tabular}

The quantities computed on the fault by ADER-DG, such as those shown in Fig. $1 \mathrm{~b}$ and $\mathrm{c}$, show generally an excellent agreement with the FEM (finite element method) reference solution, despite the asymmetric unstructured mesh surrounding the fault in the ADER-DG simulation. However, despite artificial damping the finite element solution shows high-frequency oscillations in slip rate amplitudes. The development of a supershear rupture front in TPV11 is well-captured by both methods, as shown in Fig. 2. The rupture time contour plot in Fig. 2a captures the acceleration of rupture velocity after supershear transition. Figure $2 b$ shows the normal stress variation caused by the interaction of the nonvertical fault with Earth's free surface, investigated e.g., by Rudnicki and Wu (1995), Dalguer et al. (2001), Ma and Archuleta (2006), Andrews et al. (2007), Ma and Beroza (2008). We find differences in the evolution of stresses after $10 \mathrm{~s}$ : the FEM solution reaches higher normal and along-dip shear stresses leading to a slight difference in slip rate. This might be due to fault and free surface interaction being handled differently by both methods.

Strong ground shaking due to free surface effects, including asymmetry between foot wall and hanging wall, can be observed in Fig. 3. Larger ground motions on the hanging wall than on the footwall are observed in natural earthquakes (e.g., Abrahamson and Somerville, 1996). The agreement between ground motions computed by ADER-DG and FEM in the vicinity of the fault is near-perfect.

\section{Heterogeneous background stress}

Tectonic loading plays a fundamental role in earthquake source dynamics and controls the size of an earthquake. Stress heterogeneities could potentially influence nucleation and arrest of a rupture (Day, 1982; Boatwright and Quin, 1986; Oglesby and Day, 2002; Ampuero et al., 2006). An open question is how small-scale fluctuations of initial stresses modulate the rupture process. As stresses in the Earth's interior cannot be measured directly, dynamic rupture simulations represent a proper tool to analyze the impact of stress heterogeneities. 

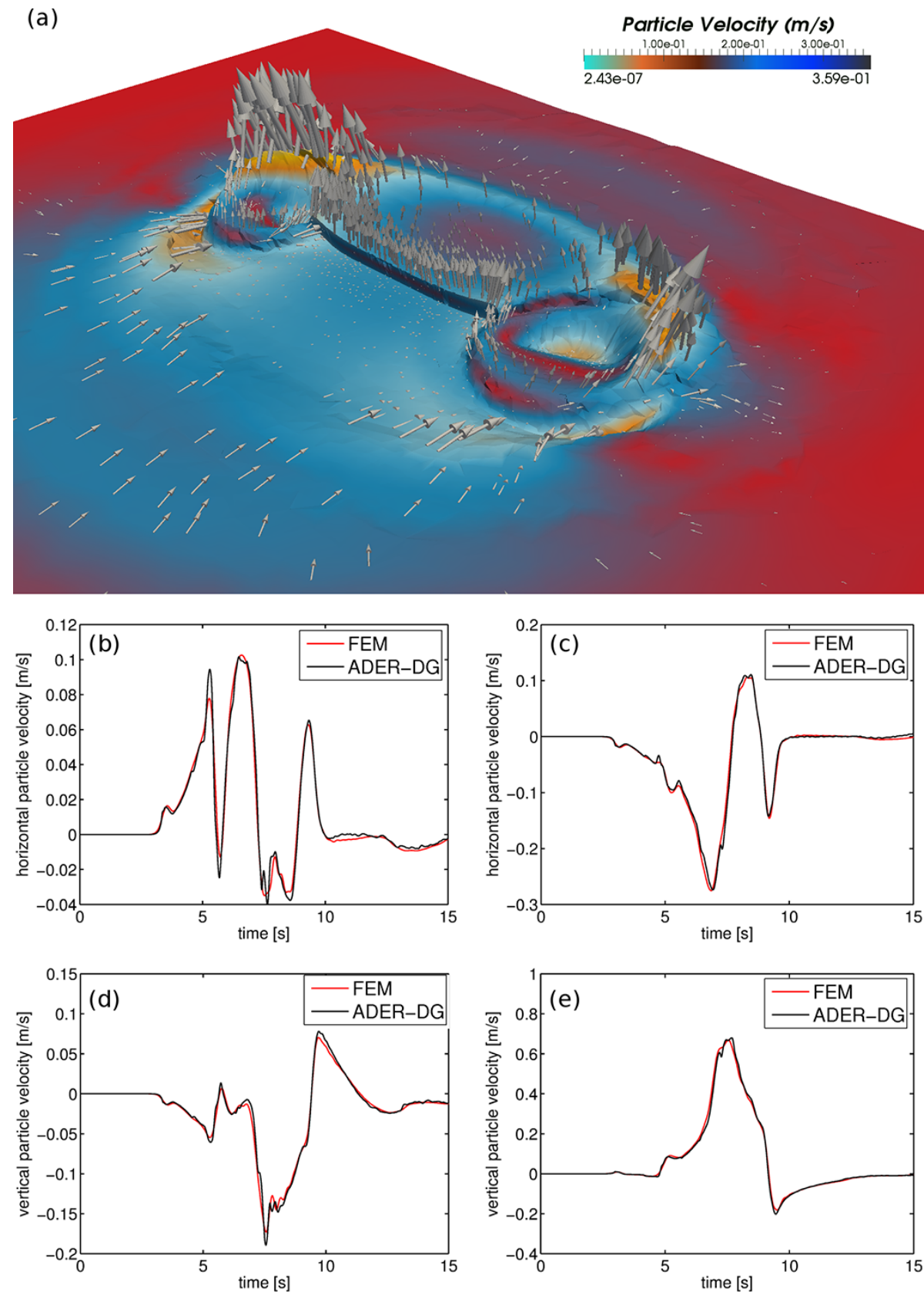

Fig. 3. (a) Asymmetric ground motion in the surrounding of the dipping fault at $t \approx 8 \mathrm{~s}$ in TPV10. The color scale indicates the magnitude of absolute particle velocity at the surface, arrows and surface morphology reflect its direction. (b) and (c) are horizontal ground velocity time series at seismic stations located $3 \mathrm{~km}$ away from the fault trace and $12 \mathrm{~km}$ along-strike on the foot wall and hanging wall, respectively. (d) and (e) are the vertical ground velocities at the same stations. The ADER-DG solution is shown in black, the FEM comparison solution in red.

The SCEC benchmark problems TPV16 and TPV17 focus on the modeling of dynamic rupture under heterogeneous initial stress conditions. Here, we consider only TPV17, as TPV16 is very similar. Randomly generated heterogeneous initial stress conditions and frictional parameters are provided as predefined input. The setup contains a planar strike-slip fault embedded in a linear elastic medium with wave speeds specified in Table 2. Linear slip-weakening governs the frictional sliding. The nucleation method combines high-stress and low- $D_{\mathrm{c}}$ values within approximately 1 and $4 \mathrm{~km}$ of the hypocenter, respectively, and a prescribed timedependent reduction of the friction coefficient that forces initially a circular rupture growth at constant speed. 
Table 2. Simulation parameters for SCEC test case TPV17.

\begin{tabular}{llr}
\hline$c_{\mathrm{P}}$ & P wave speed & $6000 \mathrm{~m} \mathrm{~s}^{-1}$ \\
$c_{\mathrm{S}}$ & Shear wave speed & $3464 \mathrm{~m} \mathrm{~s}^{-1}$ \\
$\rho$ & Density & $2670 \mathrm{~kg} \mathrm{~m}^{-3}$ \\
$A_{\text {fault }}$ & Faulting area & $48.0 \mathrm{~km} \times 19.5 \mathrm{~km}$ \\
$h$ & Element edge length & $200 \mathrm{~m}$ \\
$\mathcal{O}$ & Spatiotemporal order of accuracy & 4 \\
\hline
\end{tabular}

The effect of heterogeneous initial stresses on dynamic rupture propagation needs particular scrutiny in high-order methods, which work more efficiently on large elements (Käser et al., 2008). Even if the dispersion requirements are sufficiently addressed by large elements and a high-order approach, yet another issue is the correct sampling of the initial stress and friction data on the fault. Pelties et al. (2010) attempted to define rules to respect material properties of a complex geological medium correctly, but we are not aware of a study addressing this issue for the initial stress conditions of a dynamic rupture simulation. As introduced in Sect. 2 and Appendix A, the fault plane is located at the interface between two adjacent elements and the flux functions on the fault are modified to satisfy the friction conditions. These flux functions are integrated with a quadrature based on $(N+2)^{2}$ GPs irregularly distributed across each triangular element face, where $N$ is the polynomial degree of the basis functions. We treat heterogeneous input data by assigning initial stress values and friction parameters to each of these GPs. Here we use trilinear interpolation to map the gridded input data on the irregularly distributed GPs. In this way, the smallest possible scale of the numerical method is exploited without decreasing the element edge length and thus subelement resolution is enabled. A resolution of element edge length of $h=200 \mathrm{~m}$ and $\mathcal{O} 4$ is applied in order to test if our proposed subsampling scheme is sufficient to capture the initial stress values and frictional parameters. Mesh coarsening with increasing distance to the fault is applied.

Figure 4 demonstrates the complexity of the rupture propagation caused by the small-scale heterogeneous background stress. Considering the complexity of the input data we find good agreement between the two compared methods. Good agreement is also confirmed for shear stress and slip rate time series at two fault locations, shown in Figs. 5 and 6. Figure 7 depicts a snapshot of the horizontal component of slip rate at $5.5 \mathrm{~s}$ across the entire fault plane and indicates the fault point considered in Fig. 5. In Fig. 5c we observe a sharp feature at $\sim 5.5 \mathrm{~s}$ in the horizontal slip rate. With the aid of Fig. 7, we can identify that this signal is due to the passage of an interface wave (Dunham, 2005). Reflection at the free surface causes a healing front that passes the receiver after the rupture front and is followed immediately by a secondary slip rate peak, whose amplitude even exceeds that of the primary rupture front. At other positions on the fault, a healing effect

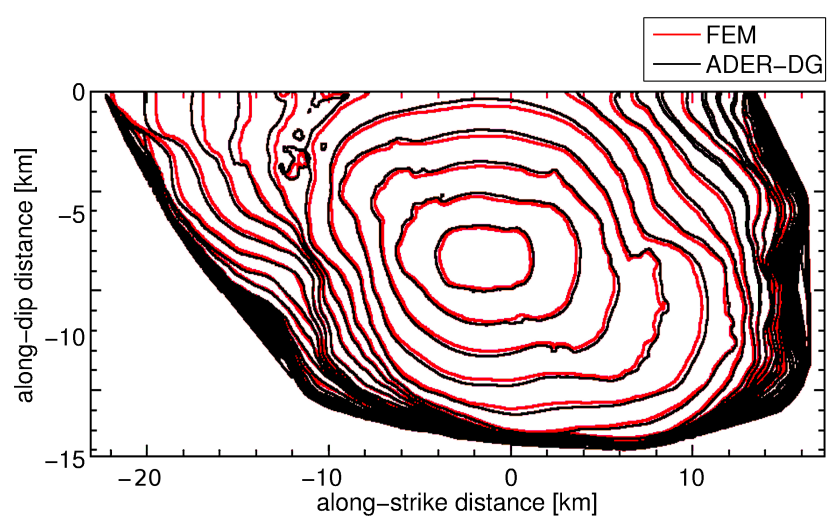

Fig. 4. Rupture front every $0.5 \mathrm{~s}$ of problem TPV17 with heterogeneous initial stress conditions. The ADER-DG rupture front is indicated in black, the FEM comparison solution in red.

caused by interface waves is not as pronounced. This feature is also resolved by other numerical methods at high resolution (see the data provided by the SCEC code comparison tool). The remarkable accuracy of the ADER-DG solver allows us to observe such features despite a relatively large element edge length of $h=200 \mathrm{~m}$. We conclude that our approach to sample input data at the Gaussian integration points leads to sufficient conformity with FaultMod results.

\section{Fault branching}

Fault branching has been observed in natural events (e.g., Schwartz et al., 2012). Incorporating the possibility of earthquake rupture propagating across multiple faults may lead to earthquake scenarios involving very different magnitudes and dynamics, which is of crucial importance for seismic hazard analysis. However, the role of fault branching in earthquake dynamics is poorly understood. On the one hand, realistic simulations need reliable information on fault geometries. On the other hand, the theoretical analysis of fault branching is limited to simplified scenarios. The path selected by a dynamic rupture beyond the junction of main fault and branch depends on the specific boundary conditions assumed at the junction (DeDontney et al., 2011).

To verify the performance of the ADER-DG algorithm on a branching fault system we discuss here SCEC's test cases TPV14 and TPV15. The geometrical setup contains two vertical, planar strike-slip faults; a main fault and a branch fault intersecting at an angle of $30^{\circ}$. The faults reach the Earth's surface. The initial stress conditions determine the fault system as right-lateral in TPV14 and as left-lateral in TPV15. Here, we focus on TPV15. 

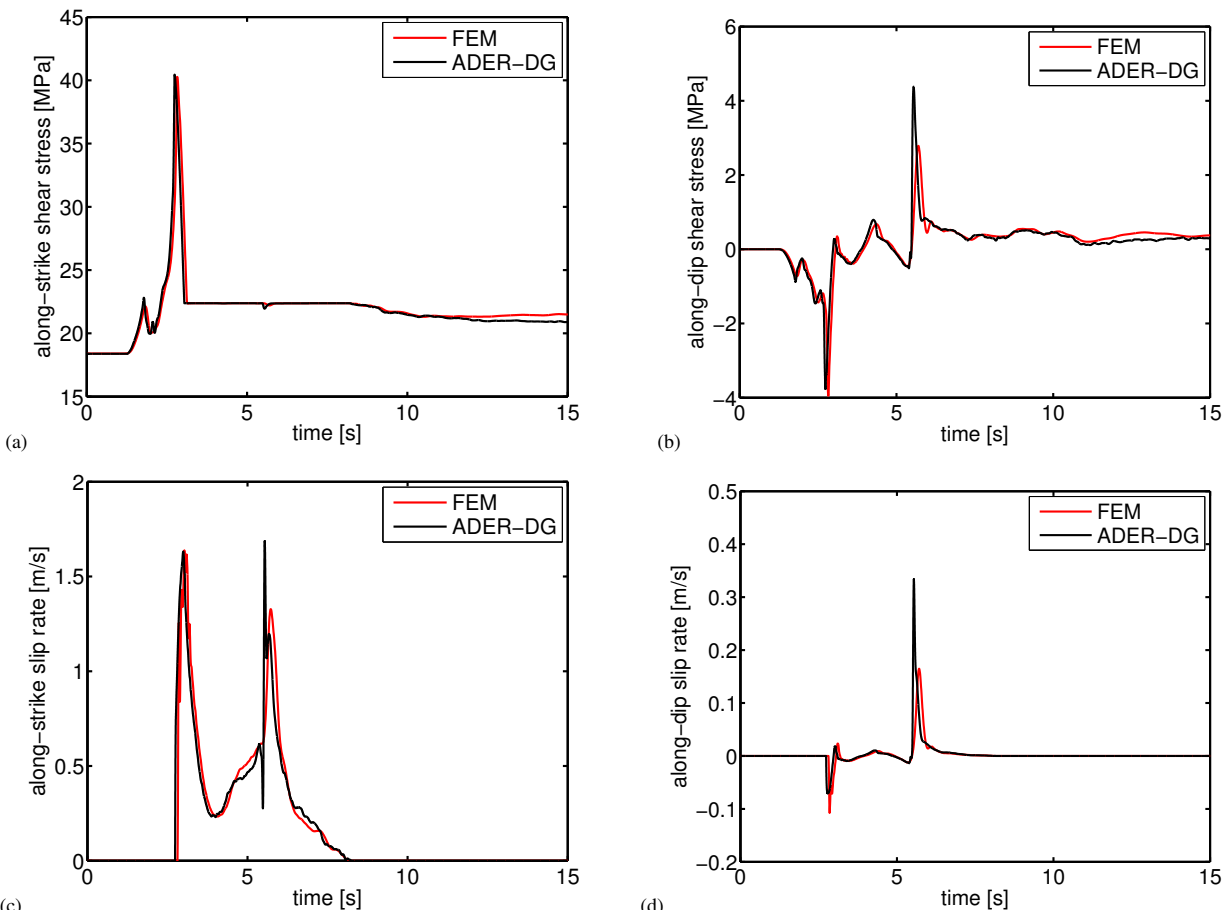

(b)

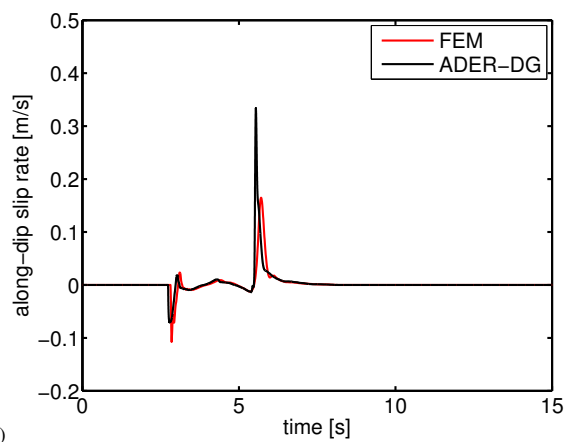

Fig. 5. Shear stresses and slip rates for TPV17 on a fault location at $9 \mathrm{~km}$ depth and $-9 \mathrm{~km}$ along strike. A sharp feature caused by an interface wave can be identified in the along-strike slip rate (c) at $t \sim 5.5 \mathrm{~s}$. The ADER-DG solution is shown in black, the FEM comparison solution in red.
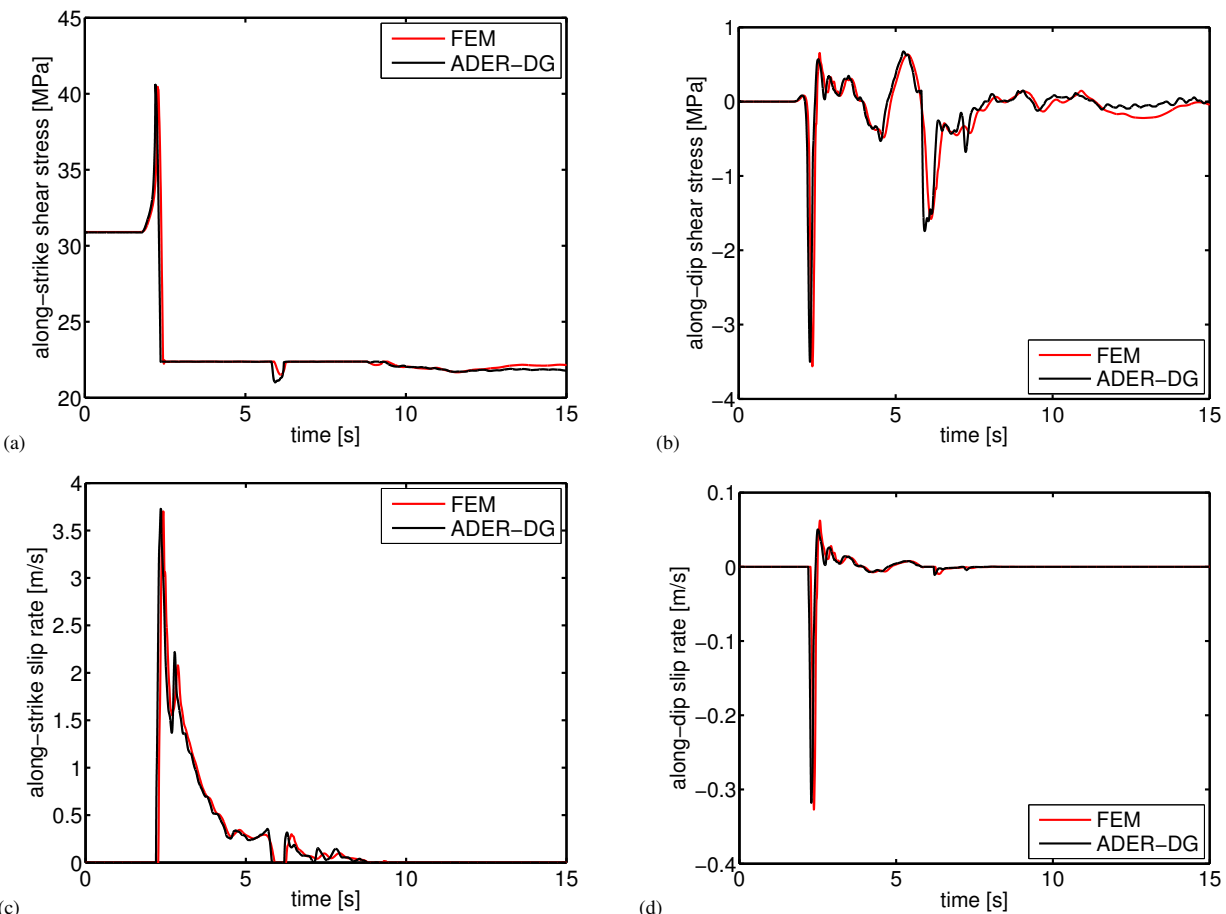

Fig. 6. Shear stresses and slip rates for TPV17 on a fault location at $9 \mathrm{~km}$ depth and $9 \mathrm{~km}$ along strike. The ADER-DG solution is shown in black, the FEM comparison solution in red. 


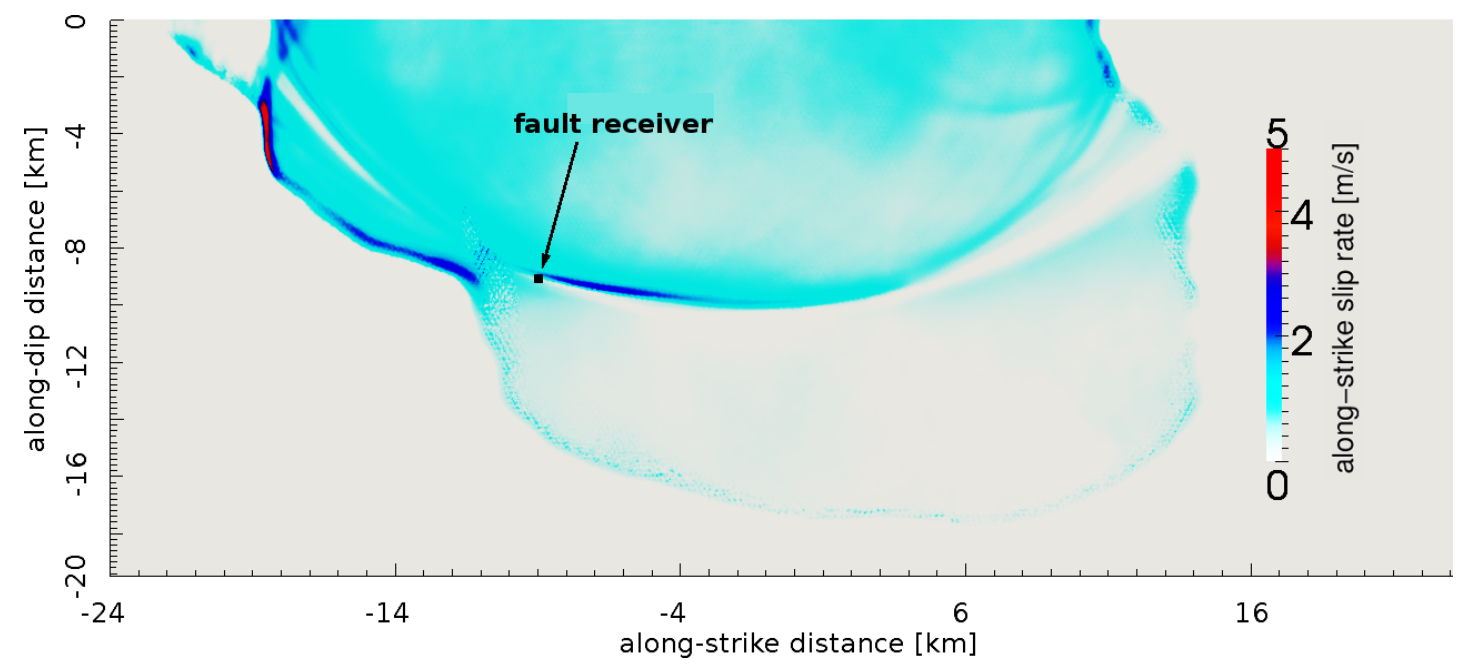

Fig. 7. Along-strike slip rate (in $\mathrm{m} \mathrm{s}^{-1}$ ) for TPV17 at $t=5.5 \mathrm{~s}$; the time at which the interface wave that originated at the free surface approaches the fault location indicated by an arrow and shown in Fig. 5. The elliptical shape of the interface wave front is clearly visible, which consists of a healing phase followed by a high slip rate front.

The intersection of main fault and branch fault is referred to as junction and is located at an along-strike distance of $0 \mathrm{~km}$. However, as specified in the benchmark description, the branch fault should not fully reach the junction. A small gap with the length of one grid spacing between the two faults is prescribed. A rupture nucleated on the main fault can propagate continuously along the main fault past the junction, but it must jump the gap to propagate onto the branch fault. Since our method can model both geometries with and without gap, we address both cases and discuss their differences. In the modal ADER-DG formulation the solution does not involve variables defined along the junction, only in the interior of the element and its faces, excluding edges and vertices. Branching benchmark problems TPV24/25 without gap at the junction were more recently formulated, but are not considered here.

We use the same mesh for the simulations with and without gap. This is achieved by either locking part of the branch (with gap) or allowing the full branch to rupture (without gap). This way, all differences in the results are only due to the influence of the gap. We choose the recommended spacing of $100 \mathrm{~m}$ as gap length, although the generally applied mesh resolution at the fault is $h=300 \mathrm{~m}$. This results in a slightly higher mesh resolution of up to $h=100 \mathrm{~m}$ in the direct vicinity of the junction. We illustrate this in a zoomed view of the junction in a 2-D setup in Fig. 8. The different colors represent different MPI partitions for parallel computing. Fault and MPI interfaces can coincide or intersect arbitrarily. Again, mesh coarsening with distance to the fault is applied. The order of accuracy in space and time is $\mathcal{O} 4$ for all simulations. We summarize the complete parameters in Table 3.
Table 3. Simulation parameters for SCEC test case TPV15.

\begin{tabular}{llr}
\hline$c_{\mathrm{P}}$ & P wave speed & $6000 \mathrm{~m} \mathrm{~s}^{-1}$ \\
$c_{\mathrm{S}}$ & Shear wave speed & $3464 \mathrm{~m} \mathrm{~s}^{-1}$ \\
$\rho$ & Density & $2670 \mathrm{~kg} \mathrm{~m}^{-3}$ \\
$\mu_{\mathrm{s}}$ & Static friction coefficient & 0.677 \\
$\mu_{\mathrm{d}}$ & Dynamic friction coefficient & 0.525 \\
$D_{\mathrm{c}}$ & Slip-weakening critical distance & $0.4 \mathrm{~m}$ \\
$\sigma_{0}$ & Background normal stress & $120.0 \mathrm{MPa}$ \\
$\tau_{0}^{\text {main }}$ & Background shear stress on main fault & $-70.0 \mathrm{MPa}$ \\
$\tau_{0}^{\text {branch }}$ & Background shear stress on branch fault & $-78.0 \mathrm{MPa}$ \\
$\tau_{0}^{\text {nuc }}$ & Nucleation shear stress along-dip & $-81.6 \mathrm{MPa}$ \\
$A_{\text {nuc }}$ & Nucleation size & $3 \mathrm{~km} \times 3 \mathrm{~km}$ \\
$A_{\text {fault }}^{\text {main }}$ & Faulting area & $28 \mathrm{~km} \times 15 \mathrm{~km}$ \\
$A_{\text {fault }}^{\text {branch }}$ & Faulting area & $12 \mathrm{~km} \times 15 \mathrm{~km}$ \\
$h$ & Element edge length & $300 \mathrm{~m}$ \\
$\mathcal{O}$ & Spatiotemporal order of accuracy & 4 \\
\hline
\end{tabular}

For comparison we choose the results of FaultMod with 100 and $50 \mathrm{~m}$ node spacing. As expected, we see that the $100 \mathrm{~m}$ results of FaultMod are closer to the ADER-DG solution with gap and FaultMod's $50 \mathrm{~m}$ results are closer to the ADER-DG without-gap solution. First, we discuss the rupture times obtained with each method and resolution shown on the main fault in Fig. 9a and on the branch in Fig. 9b. We find very good agreement in the early rupture evolution. Clear differences occur along the junction: whereas in both FaultMod simulations rupture stops shortly after the junction, except for some slip at the fault bottom and at the free surface, in the ADER-DG simulation with gap, rupture continues further along the main fault. In the ADER-DG solution without gap rupture continues only for a short distance along the main fault, stops there a bit earlier than in the three other solutions, but exhibits early rupture near the junction. This 
observation can be linked to earlier rupture initiation at the branch (see Fig. 9b). The branch rupture of the ADER-DG solution with gap starts slightly later, similar to the FaultMod solutions, as the gap needs to be overcome first. With increasing distance along strike, the rupture in the ADERDG solution with gap propagates slower than in all other solutions, however, only along the branch. The concentration of rupture fronts at an along-strike distance $>0 \mathrm{~km}$ on the main fault for the ADER-DG simulation without gap (concentrated blue lines in Fig. 9a) results from a smooth, spontaneous rupture arrest in the branch (as opposed to abrupt arrest by a barrier). Minor differences of rupture time occur at the end of the seismogenic zone and at the free surface along the branching fault.

In Fig. 10 we present time series of shear stresses and slip rates in strike and dip direction on the branch at $9 \mathrm{~km}$ alongstrike from the junction and $7.5 \mathrm{~km}$ depth. In general, we observe a good agreement between the four solutions. Only small differences in the dip components (Fig. 10b, d) are visible at $\sim 7.5 \mathrm{~s}$. Since the dip component is one order of magnitude smaller than the strike component, these differences are considered negligible.

Larger discrepancies can be observed closer to the junction, for example on the main fault at $2 \mathrm{~km}$ along-strike from the junction and $7.5 \mathrm{~km}$ depth, as shown in Fig. 11. Whereas the along-dip shear stress (Fig. 11b) and the normal stress (Fig. 11c) are very similar, the along-strike shear stresses differ clearly in all four simulations. The $100 \mathrm{~m}$ results of FaultMod are closer to the ADER-DG solution with gap and FaultMod's $50 \mathrm{~m}$ results are closer to the ADER-DG solution without gap. We argue that this is expected, as the geometrical setups are more similar. In general, solutions far away from the junction and off the fault (not shown here) match very well. We find discrepancies only in the direct vicinity of the junction, which can be traced back to the gap between main and branching faults. DeDontney et al. (2011) demonstrated that small differences in fault geometry, including the details at fault junctions, can lead to different rupture paths, which calls for caution in the design and interpretation of simulations of rupture branching scenarios.

\section{Bimaterial faults}

Natural faults often separate rocks with different material properties (e.g., Thurber et al., 2006), which leads to normal stress variations during faulting that influence dynamic rupture propagation (Harris and Day, 1997; Ampuero and Ben-Zion, 2008; Brietzke et al., 2009). Such stress perturbations can generate specific rupture patterns, for example selfsustaining pulses with a preferred rupture direction, and are therefore of particular interest. The instantaneous response of shear strength to normal stress changes (Coulomb friction) at bimaterial interfaces leads to an ill-posed problem for a wide range of elastic material contrasts, causing an instability at

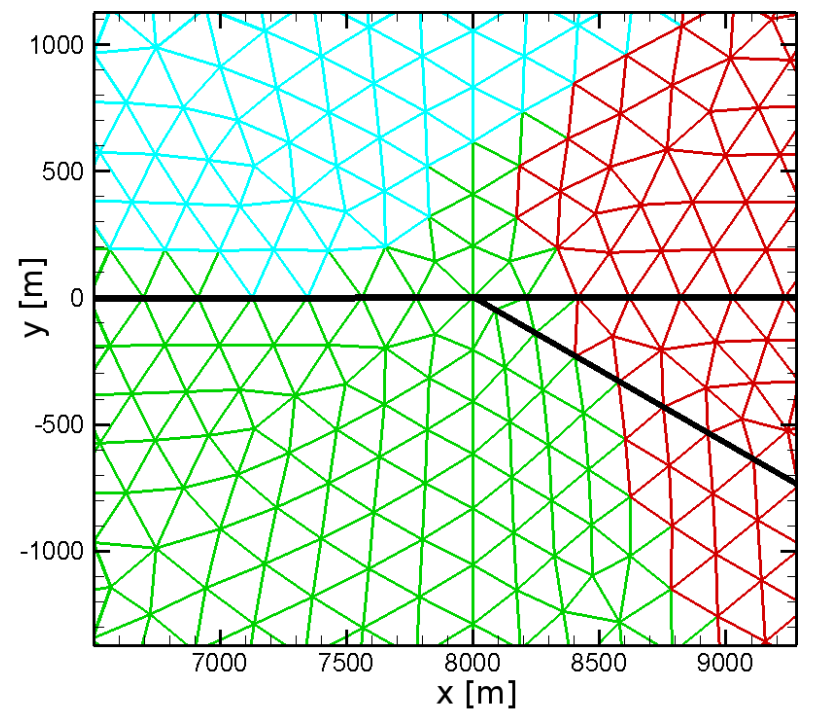

Fig. 8. Zoom view of the junction of TPV15 (2-D example) to illustrate the parallelization concept and discretization strategy.

all wavelengths (Adams, 1995). Convergence through gridsize reduction cannot be achieved in ill-posed problems and regularization needs to be applied. We address regularization concerns in Appendix B.

Here, we concentrate on the well-posed bimaterial benchmark case TPV6, which is specified to be conducted without any form of regularization. We define a near side and a far side of the fault plane. The far side has a wave speed reduction of $60 \%$ and a density reduction of $20 \%$. The fault is a planar strike-slip fault that reaches the free surface. Frictional sliding is governed by linear slip-weakening. Model parameters can be found in Table 4. The ADER-DG results are computed with $h=200 \mathrm{~m}$ and $\mathcal{O} 4$ for space and time and without viscous damping. FaultMod uses a $50 \mathrm{~m}$ grid spacing, and applies Newmark damping and a viscous layer surrounding the fault to suppress artificial oscillations.

Figure 12 shows the evolution of the rupture front. We find very good agreement of rupture speeds between the two methods. Only small differences in the arrival time occur in the along-dip direction. These differences can be noted as well in the time series of velocity and stress at a location at the surface on the near side of the fault, above the nucleation zone, shown in Fig. 13. Whereas the initial waves arrive simultaneously for both methods, the FEM solution evolves slower to the peak values, inducing a delay in rupture times relative to the ADER-DG solution. This effect might be caused by the damping algorithm or the viscosity layer surrounding the fault implemented in FaultMod. The general temporal evolutions of stress and particle velocity are, however, very similar. Only the relatively small, vertical components of shear stress and velocity show larger deviations as observable in Fig. 13b and e. The match of the normal components is again very good, although FaultMod 


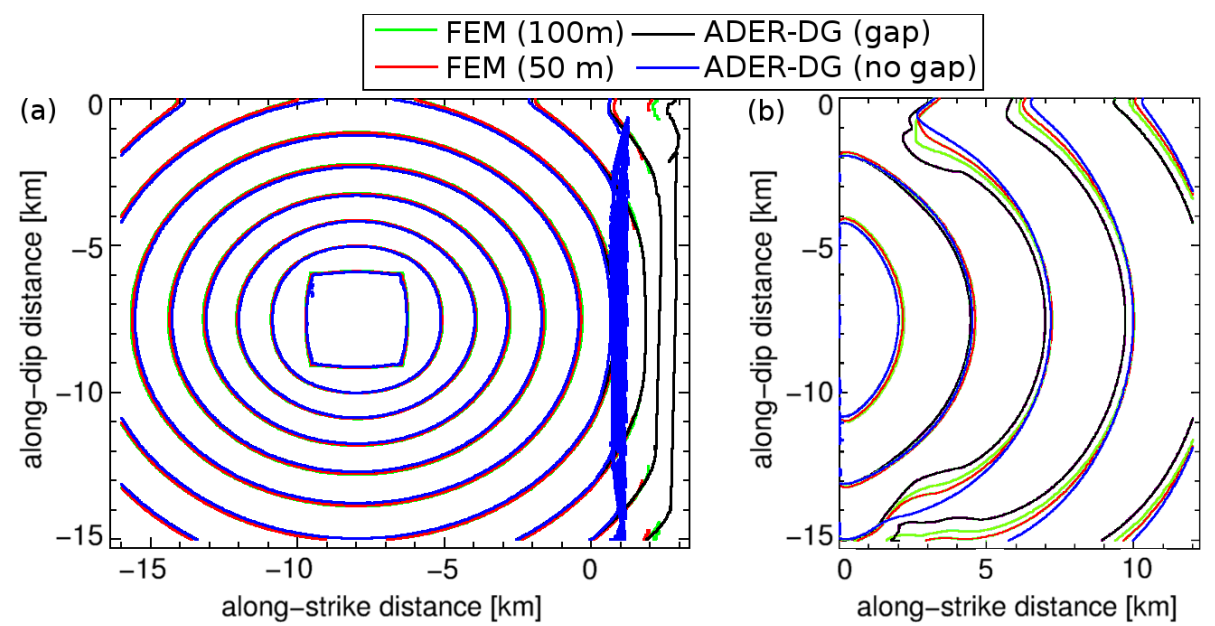

Fig. 9. Rupture time contours of benchmark TPV15 on (a) the main fault and (b) the branch fault. The junction is located at the along-strike distance of $0 \mathrm{~km}$. ADER-DG solutions are shown in black (with gap) and blue (without gap), the FEM comparison solutions in red (50 $\mathrm{m}$ discretization) and green (100 $\mathrm{m}$ discretization).
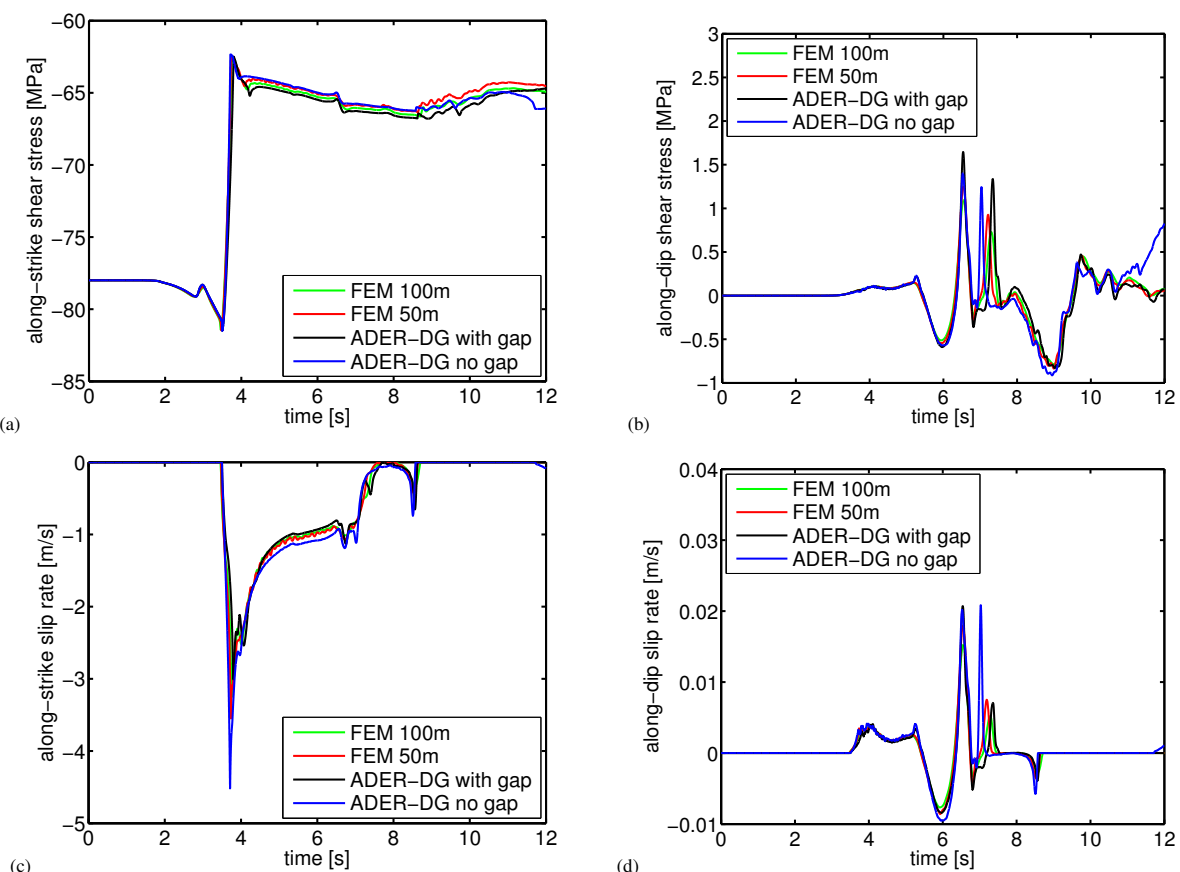

Fig. 10. Shear stresses and slip rates for TPV 15 on the branch fault at $9 \mathrm{~km}$ along-strike and $7.5 \mathrm{~km}$ depth. ADER-DG solutions are shown in black (with gap) and blue (without gap), the FEM comparison solutions in red (50 m discretization) and green (100 m discretization).

yields slightly smaller peak values (Fig. 13c, f). Note that the ADER-DG solution exhibits only small oscillations in all time series. Time series recorded at the far side of the fault, at larger distance to the nucleation zone, and at $7.5 \mathrm{~km}$ depth are presented in Fig. 14. At this location, rupture arrival time and slip rate peak match better than for the near-side station above the nucleation zone. We conclude that for the wellposed bimaterial problem a very good agreement between ADER-DG and FaultMod is reached.

\section{Rate- and state-dependent friction}

The appropriate form of the constitutive law that describes the relationship between fault stress and slip along a fault plane is a topic of intense research. Widely applied empirical friction laws are derived from small-scale laboratory experiments (Brace and Byerlee, 1966; Ruina, 1983; Ohnaka and Kuwahara, 1990; Di Toro et al., 2005; Niemeijer et al., 2010), although the question of a proper scaling of the parameters 


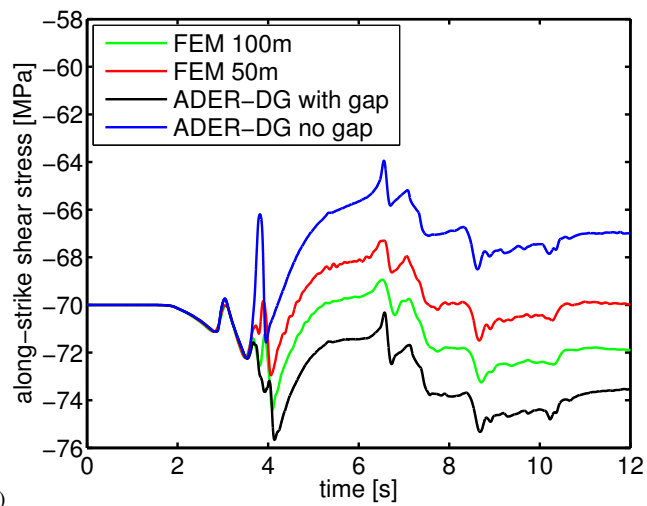

(a)

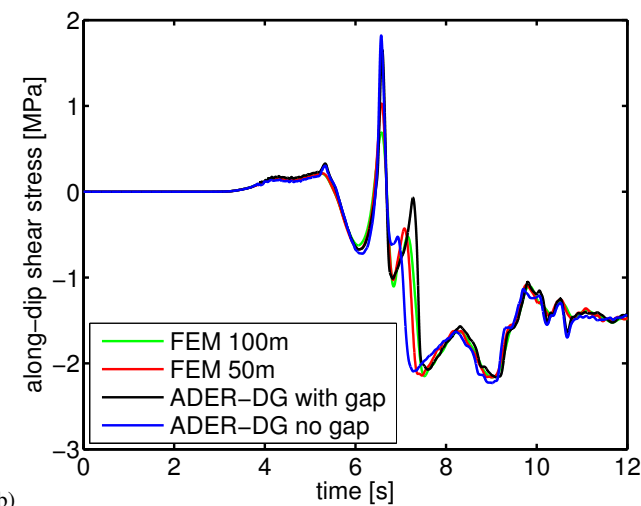

(b)

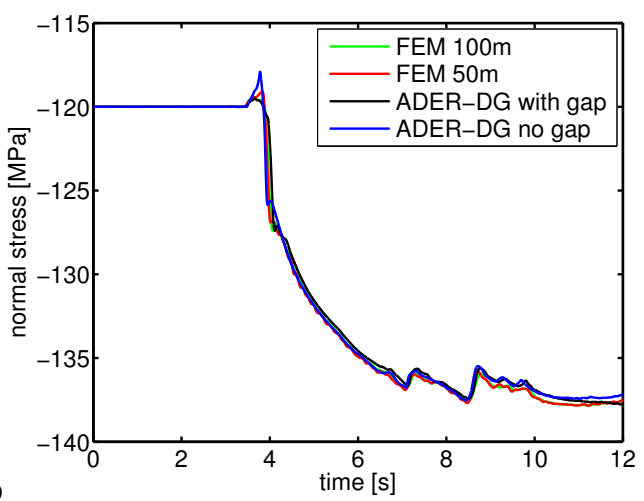

Fig. 11. Shear and normal stresses of TPV15 recorded on the main fault at $2 \mathrm{~km}$ along-strike from the junction and $7.5 \mathrm{~km}$ depth. Differences are due to differences in fault geometry at the junction as discussed in the main text. ADER-DG solutions are shown in black (with gap) and blue (without gap), the FEM comparison solutions in red (50 $\mathrm{m}$ discretization) and green (100 $\mathrm{m}$ discretization).

to seismic faulting is often evoked. Friction laws differ in the choice of primary variables that control fault weakening: cumulated slip (slip-weakening friction), slip velocity (ratedependent friction) and/or average age of the microstructural contacts or other state variables (state-dependent friction). At high slip rates, physical weakening processes involving thermal coupling, as for example thermal pressurization of pore fluids, flash heating or melting, are thought to cause a
Table 4. Simulation parameters for SCEC test case TPV6.

\begin{tabular}{llr}
\hline$\rho_{\text {near }}$ & Density & $2670 \mathrm{~kg} \mathrm{~m}^{-3}$ \\
$c_{P, \text { near }}$ & P wave speed & $6000 \mathrm{~m} \mathrm{~s}^{-1}$ \\
$c_{\mathrm{S} \text {,near }}$ & Shear wave speed & $3464 \mathrm{~m} \mathrm{~s}^{-1}$ \\
$\rho_{\text {far }}$ & Density & $2225 \mathrm{~kg} \mathrm{~m}^{-3}$ \\
$c_{P, \text { far }}$ & P wave speed & $3750 \mathrm{~m} \mathrm{~s}^{-1}$ \\
$c_{\mathrm{S} \text {,far }}$ & Shear wave speed & $2165 \mathrm{~m} \mathrm{~s}^{-1}$ \\
$\mu_{\mathrm{S}}$ & Static friction coefficient & 0.677 \\
$\mu_{\mathrm{d}}$ & Dynamic friction coefficient & 0.525 \\
$D_{\mathrm{c}}$ & Slip-weakening critical distance & $0.4 \mathrm{~m}$ \\
$\sigma_{0}$ & Background normal stress & $120.0 \mathrm{MPa}$ \\
$\tau_{0}$ & Background shear stress on main fault & $70.0 \mathrm{MPa}$ \\
$\tau_{0}^{\text {nuc }}$ & Nucleation shear stress along-dip & $81.6 \mathrm{MPa}$ \\
$A_{\text {nuc }}$ & Nucleation size & $3 \mathrm{~km} \times 3 \mathrm{~km}$ \\
$A_{\text {fault }}$ & Faulting area & $30 \mathrm{~km} \times 15 \mathrm{~km}$ \\
$h$ & Element edge length & $200 \mathrm{~m}$ \\
$\mathcal{O}$ & Spatiotemporal order of accuracy & 4 \\
\hline
\end{tabular}

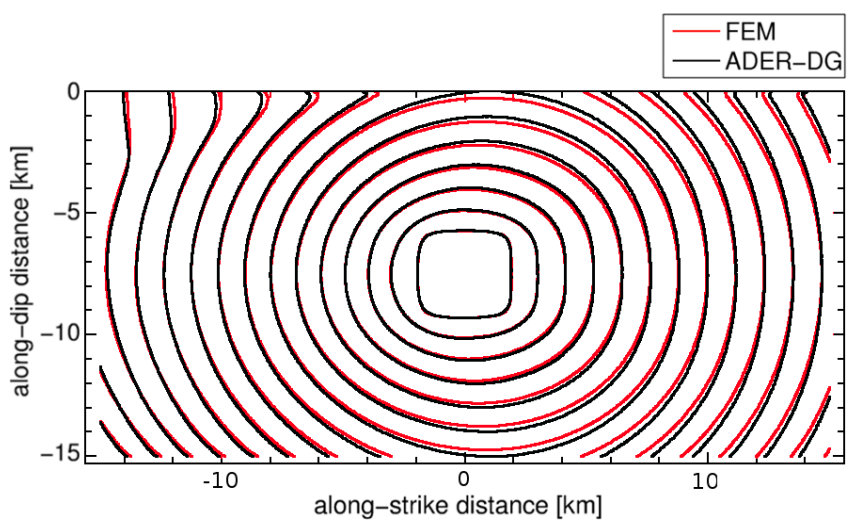

Fig. 12. Rupture front every $0.5 \mathrm{~s}$ in problem TPV6 with bimaterial interface. The ADER-DG solution is shown in black, the FEM comparison solution in red.

dramatic decrease of effective friction as function of slip velocity, referred to as fast velocity-weakening.

The implementation of slip-weakening friction in the ADER-DG algorithm has been presented in de la Puente et al. (2009); Pelties et al. (2012). Here we present and verify the implementations of rate- and state-dependent friction.

\subsection{Slow velocity friction}

Let the frictional strength of the fault evolve as follows:

$\tau_{\mathrm{S}}=\sigma\left(\mu_{0}+a \ln \frac{V}{V_{0}}+b \ln \frac{V_{0} \Theta}{L}\right)$,

where $\sigma$ is the (constant) effective normal stress, $L$ is a characteristic slip scale and $\mu_{0}$ is the reference value of the friction coefficient at steady-state sliding at reference velocity $V_{0}$. The rate-dependent term is thought to reflect a thermally activated Arrhenius process involving the failure of atomic bonds at microcontacts of the sliding surfaces (Rice et al., 2001). The state-dependent term is thought to reflect the 

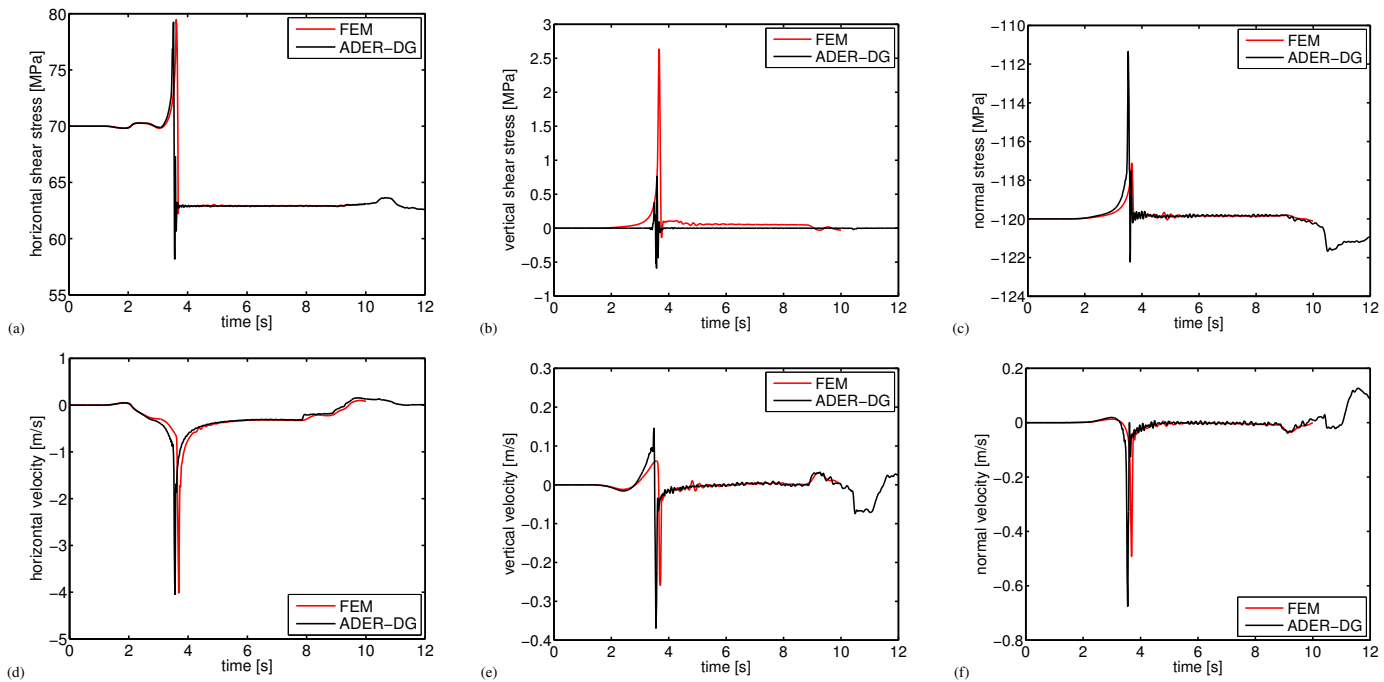

Fig. 13. Stresses and velocities of TPV6 at a surface location on the near side of the fault at $0 \mathrm{~km}$ along-strike and above the hypocenter. The ADER-DG solution is shown in black, the FEM comparison solution in red.
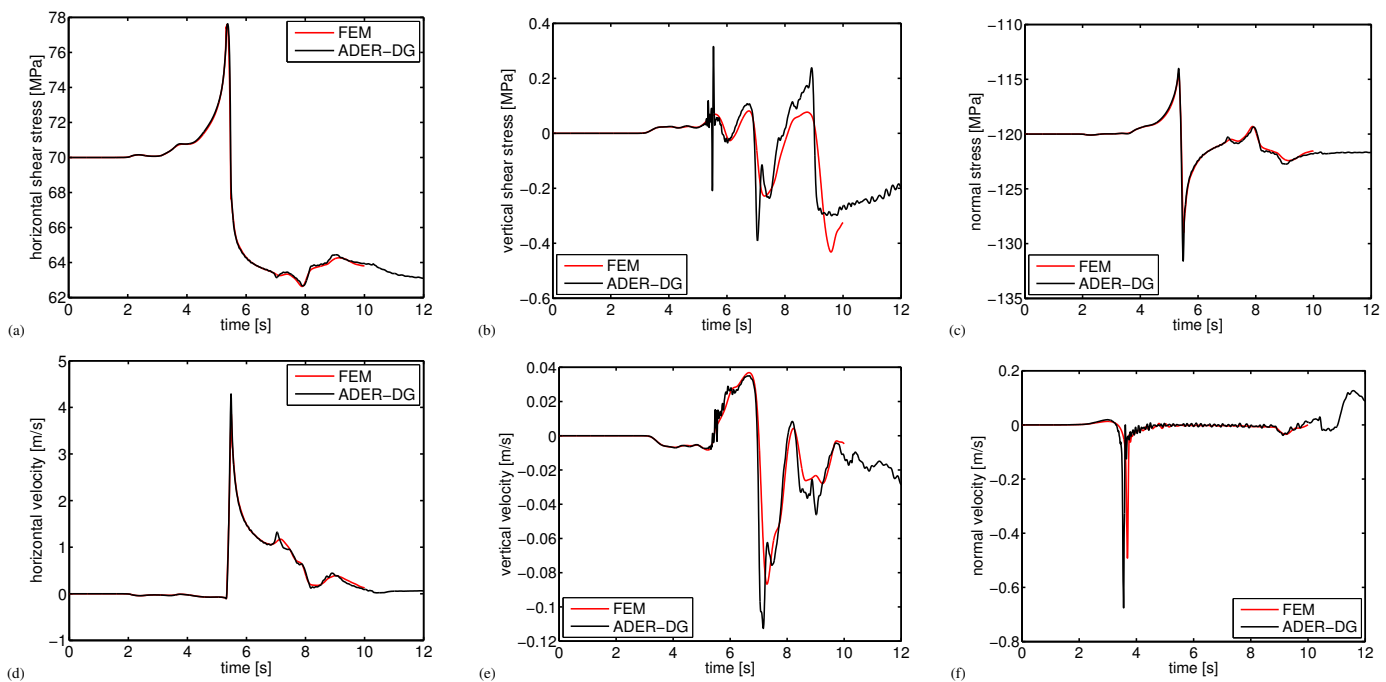

Fig. 14. Stresses and velocities of TPV6 at a location on the far side of the fault at $-12 \mathrm{~km}$ along-strike and $7.5 \mathrm{~km}$ depth, which corresponds to the hypocenter depth. The ADER-DG solution is shown in black, the FEM comparison solution in red.

product of the true contact area and the intrinsic strength of those contacts. In the ageing law formulation the state variable evolves as

$\dot{\Theta}=1-V \frac{\Theta}{L}$,

whereas in the slip law formulation it is

$\dot{\Theta}=-V \frac{\Theta}{L} \ln V \frac{\Theta}{L}$.

The implementation of rate- and state-dependent friction follows in principle Kaneko et al. (2008). The fault stresses in the Godunov state in ADER-DG play the same role as the "stick tractions" in the spectral element method (see Appendix A). First, we update the state variable using the evolution laws, Eqs. (2) or (3) based on slip rate and state variable values obtained in the previous time step as initial guess. Next, a five-stage Newton-Raphson algorithm is employed to determine the slip rate. Starting from the average slip rate between the initial guess and the one newly evaluated we repeat the entire update scheme a second time. This procedure is executed in every sub-time step of the ADER integration scheme (de la Puente et al., 2009; Pelties et al., 2012).

We benchmark the ADER-DG scheme performance for the ageing law in the SCEC test case TPV101. We model a planar fault in an isotropic, linear elastic half-space. 
Table 5. Simulation parameters for SCEC test case TPV101.

\begin{tabular}{|c|c|c|}
\hline$c_{\mathrm{P}}$ & P wave speed & $6000 \mathrm{~m} \mathrm{~s}^{-1}$ \\
\hline$c_{\mathrm{S}}$ & Shear wave speed & $3464 \mathrm{~m} \mathrm{~s}^{-1}$ \\
\hline$\rho$ & Density & $2670 \mathrm{~kg} \mathrm{~m}^{-3}$ \\
\hline$\mu_{0}$ & Reference friction coefficient TPV10 & 0.6 \\
\hline$V_{0}$ & Reference slip velocity & $10^{-6} \mathrm{~m} \mathrm{~s}^{-1}$ \\
\hline$a(x, y)$ & Frictional evolution coefficient & $0.008+\Delta a(x, y)$ \\
\hline$b$ & Frictional state coefficient & 0.012 \\
\hline$L$ & characteristic slip scale & $0.02 \mathrm{~m}$ \\
\hline$V_{\text {ini }}$ & Initial sliding velocity & $10^{-12} \mathrm{~m} \mathrm{~s}^{-1}$ \\
\hline$\Theta_{\text {ini }}$ & Initial state variable & $1.60624 \times 10^{9} \mathrm{~s}+\Delta \Theta(x, y)$ \\
\hline$\tau_{0}$ & Background shear stress along-strike & $75 \mathrm{MPa}$ \\
\hline$\sigma_{0}$ & Background normal stress & $120 \mathrm{MPa}$ \\
\hline$\Delta \tau_{0}$ & Nucleating background shear stress perturbation & $25 \mathrm{MPa}$ \\
\hline$r_{\text {nuc }}$ & Nucleation radius & $3 \mathrm{~km}$ \\
\hline$t_{\text {nuc }}$ & Nucleation time & $1 \mathrm{~s}$ \\
\hline$\left(x_{0}, y_{0}\right)$ & Hypocenter & $(0,7.5 \mathrm{~km})$ \\
\hline$A_{\text {fault }}$ & Velocity-weakening faulting area & $30 \mathrm{~km} \times 15 \mathrm{~km}$ \\
\hline$h$ & Element edge length & $250 \mathrm{~m}$ \\
\hline$O$ & Spatiotemporal order of accuracy & 4 \\
\hline
\end{tabular}
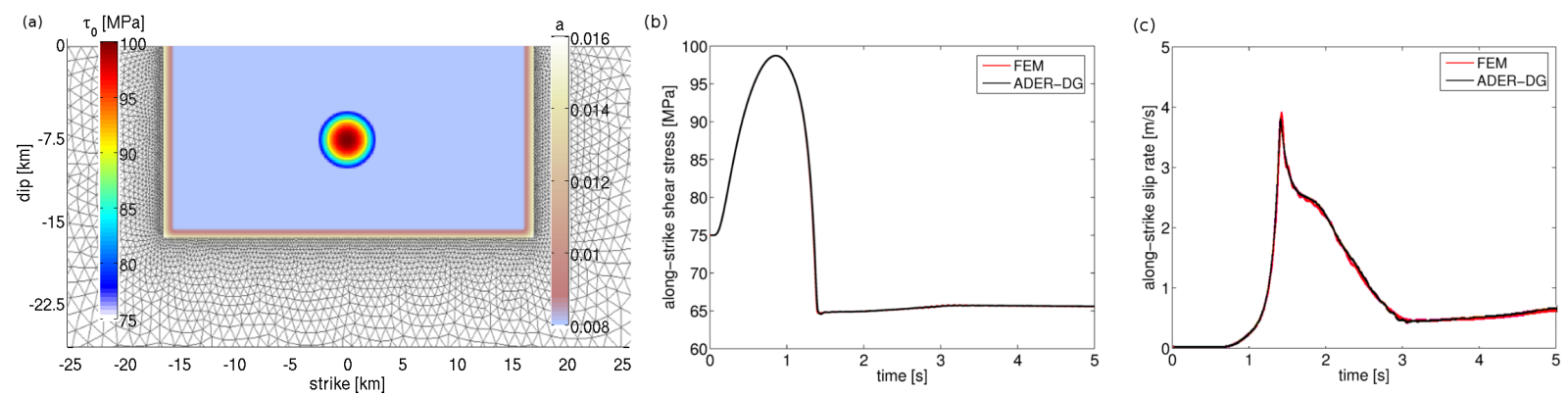

Fig. 15. (a) Nucleation and initial frictional parameters of SCEC test case TPV101. A velocity-weakening fault smoothly transitions to a surrounding velocity-strengthening material, by adapting the frictional parameter $a$. Nucleation is achieved by prescribing a space- and time-dependent circular stress perturbation. Along-strike (b) shear stress and (c) slip rate during nucleation at the hypocenter, located at $0 \mathrm{~km}$ along-strike and $7.5 \mathrm{~km}$ along-dip. The ADER-DG solution is shown in black, the FEM comparison solution in red.

The model parametrization is given in Table 5. A transition layer of $3 \mathrm{~km}$ width in which the frictional properties continuously change from velocity-weakening to velocitystrengthening surrounds the central velocity-weakening region of the fault. Outside of the transition region, the fault is velocity-strengthening. The friction-law parameter $a$ (and thus, for self-consistency, the initial state variable) is space dependent, but the initial velocity and normal and horizontal shear stresses are uniform along the fault plane. The medium on the two sides of the fault is initially moving with equal and opposite horizontal velocities of $V_{0} / 2$. Rupture is nucleated by imposing a horizontal shear traction perturbation that grows smoothly in time and space to its maximum amplitude $\Delta \tau_{0}$ over a finite time interval $T$ in a region of the fault of radius $R$. Figure 15a shows the initial shear stress and the lateral mesh coarsening around the fault plane. Figure $15 \mathrm{~b}$ and c illustrate the near-perfect agreement of ADER-DG and
FEM in along-strike shear stress and slip rate at the hypocenter throughout the nucleation period.

\subsection{Fast velocity-weakening}

In the same manner, we implement a rate- and statedependent friction law with fast velocity-weakening at slip rates higher than a characteristic velocity, as adopted by Ampuero and Ben-Zion (2008), Dunham et al. (2011) and Gabriel et al. (2012, 2013). Strong velocity-weakening has been proposed to fit results of laboratory experiments at fast slip velocities (see e.g., Di Toro et al., 2011 and references therein) and is predicted by a flash heating model (Rice, 2006). The frictional strength is determined by the slip velocity $(V)$ and a state variable $(\Theta)$ as

$$
\tau_{\mathrm{S}}=\sigma\left(a \operatorname{arcsinh}\left[\frac{V}{2 V_{0}} \exp \frac{\Theta}{a}\right]\right),
$$



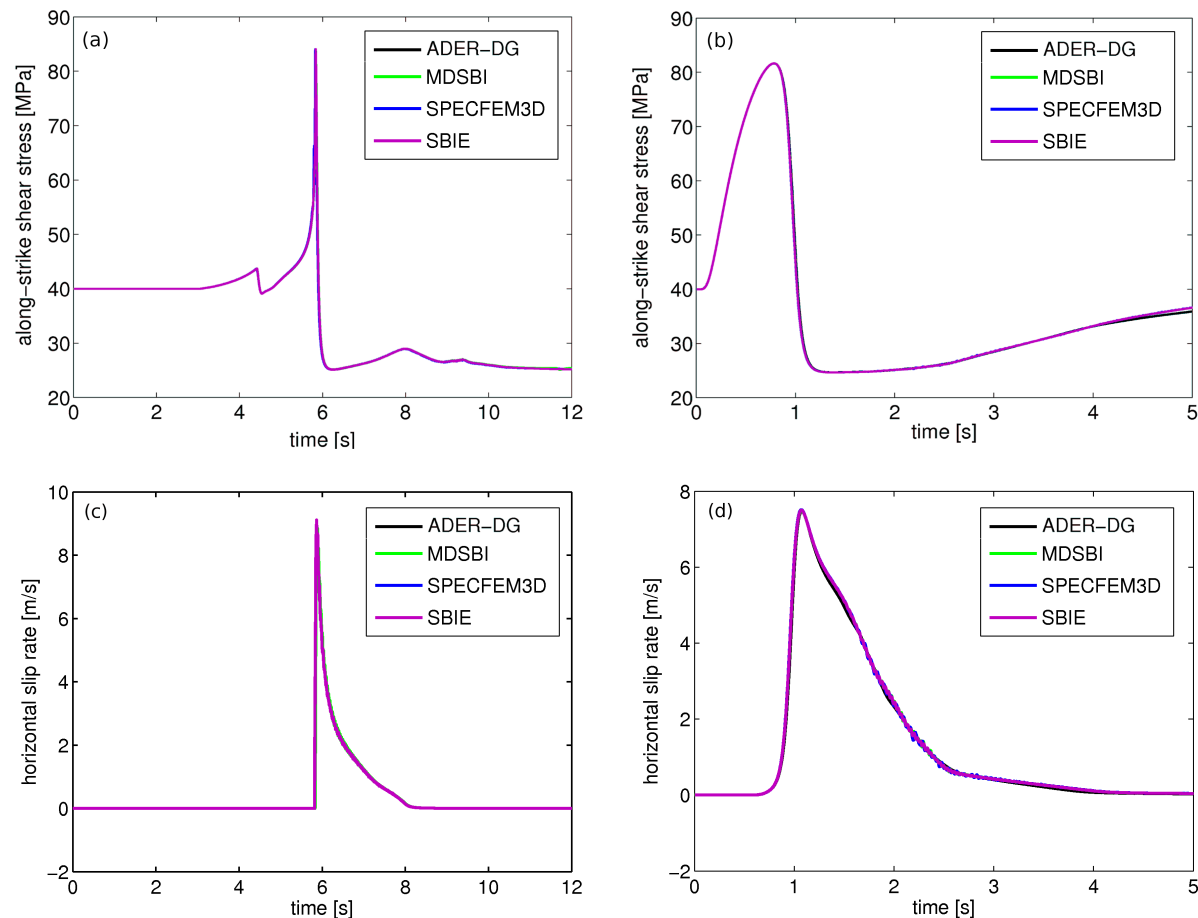

Fig. 16. Fast velocity-weakening benchmark TPV103 results. Comparison of ADER-DG (black) with the high-order dynamic rupture software packages MDSBI, SPECFEM3D and a SBIE implementation. Along-strike (a) shear stress and (c) slip rate on the fault at $12 \mathrm{~km}$ along-strike and $3 \mathrm{~km}$ along-dip. Along-strike (b) shear stress and (d) slip rate during nucleation at the hypocenter at strike $0 \mathrm{~km}$, dip $7.5 \mathrm{~km}$. The ADER-DG solution is shown in black, the comparison solutions of MDSBI in green, SPECFEM3D in blue and SBIE in purple.

Table 6. Simulation parameters for SCEC test case TPV103, which differ from test case TPV101.

\begin{tabular}{llr}
\hline$a(x, y)$ & Frictional evolution coefficient & $0.01+\Delta a(x, y)$ \\
$b$ & Frictional state coefficient & 0.014 \\
$L$ & Characteristic slip scale & $0.4 \mathrm{~m}$ \\
$V_{\mathrm{W}}$ & Weakening sliding velocity & $0.1+\Delta V_{\mathrm{W}}(x, y) \mathrm{m} \mathrm{s}^{-1}$ \\
$\mu_{\mathrm{W}}$ & Weakening friction coefficient & 0.2 \\
$V_{\mathrm{ini}}$ & Initial sliding velocity & $10^{-16} \mathrm{~m} \mathrm{~s}^{-1}$ \\
$\Theta_{\text {ini }}$ & Initial state variable & $0.5636 \mathrm{~s}+\Delta \Theta(x, y)$ \\
$\tau_{0}$ & Background shear stress along-strike & $40 \mathrm{MPa}$ \\
$\Delta \tau_{0}$ & Nucleating background shear stress perturbation & $45 \mathrm{MPa}$ \\
$A_{\text {fault }}$ & Velocity-weakening faulting area & $30 \mathrm{~km} \times 15 \mathrm{~km}$ \\
$h$ & Element edge length & $250 \mathrm{~m}$ \\
$O$ & Spatiotemporal order of accuracy & 4 \\
\hline
\end{tabular}

where $a$ is a positive coefficient quantifying a direct effect and $V_{0}$ is a reference slip rate. The state variable has units of slip and obeys the following evolution equation:

$\dot{\Theta}=-\frac{V}{L}\left(\Theta-a \ln \left[\frac{2 V_{0}}{V} \sinh \left\{\frac{\mu_{\mathrm{ss}}}{a}\right\}\right]\right)$.

Following Noda et al. (2009), we regularize the steadystate friction coefficient $\mu_{\mathrm{ss}}$ (obtained if $\dot{\Theta}=0$ ) in the framework of rate-and-state friction in the slip law form
$\mu_{\mathrm{ss}}(V)=\mu_{\mathrm{s}}+\frac{\mu_{0}-(b-a) \ln \left(\frac{V}{V_{0}}\right)-\mu_{\mathrm{w}}}{\left(1+\left[\frac{V}{V_{\mathrm{w}}}\right]^{8}\right)^{1 / 8}}$,

with $\mu_{\mathrm{s}}$ being the static friction coefficient, $\mu_{0}$ a reference friction coefficient, $V_{\mathrm{w}}$ a weakening velocity scale, $\mu_{w}$ the fully weakened friction coefficient and $b$ a positive coefficient quantifying an evolution effect. In this formulation, the transition between low velocity friction and strongly 
velocity-weakening friction is relatively smooth, which is favorable for numerical accuracy (Dunham et al., 2011).

We perform SCEC test case TPV103, which differs from the slow velocity friction test case TPV101 as summarized in Table 6. In Fig. 16 we show near-perfect agreement of ADER-DG with other high-order dynamic rupture software packages based on a variety of numerical methods: the multi-dimensional spectral boundary integral code MDSBI (Dunham, 2008), the three-dimensional spectral elements code SPECFEM3D (Kaneko et al., 2008) and the three-dimensional spectral boundary integral element (SBIE) method implementation by Lapusta and Liu (2009). The agreement of the modeled dynamic rupture processes is illustrated by the along-strike shear stress and slip rate on the fault outside the nucleation zone (Fig. 16a, c) and at the hypocenter (Fig. 16b, d).

\section{Conclusions}

We show the successful application of an ADER-DG scheme to advanced dynamic rupture test scenarios. Its performance is verified by comparison to the established finite element method FaultMod in various faulting setups, including complex geometries and heterogeneity of physical model parameters across the fault. The implementation of rate- and statedependent constitutive relationships is additionally benchmarked against other high-order dynamic rupture codes.

All time series computed with the ADER-DG method presented here are raw and unfiltered. Due to the properties of the exact Riemann solver, the solutions on the fault remain free of spurious oscillations even under complex geometric and physical conditions.
We discuss some specific properties and outcomes of studying dynamic rupture with the ADER-DG method: the resolution of small-scale interface waves on a heterogeneously prestressed fault, the smoothness of results at a bimaterial fault without experimentally motivated regularization, and the possible impact of a gap at the junction of a fault branching system.

The method is specifically suited for dynamic rupture problems on complex fault geometries by facilitating mesh refinement and coarsening. Mesh resolution can be adapted to ensure fine sampling of the cohesive zone on the fault, while satisfying the dispersion requirements of pure wave propagation away from the fault. Accurate propagation over distances of many wavelengths is enabled by a high-order accurate discretization of the wave equation. Furthermore, a smooth resolution of frictional sliding with minimized numerical artifacts is obtained, ensuring physical reliability of the results.

We conclude that the advanced geometric flexibility of SeisSol combined with its enhanced accuracy positions it as a competitive tool to study earthquake dynamics in complicated setups. 


\section{Appendix A}

\section{Integration of fault dynamics across dissimilar material interfaces into the ADER-DG algorithm}

De la Puente et al. (2009) and Pelties et al. (2012) elaborated the incorporation of fault dynamics in homogeneous materials into the ADER-DG framework. Here, we describe a general algorithm for implementing fault dynamics in methods using flux functions, which is also applicable for faults that separate rocks with different material properties. Our presentation disregards the specifics of the ADER-DG method. In particular, the algorithm is independent of the chosen timeintegration scheme. To this end, the algorithm might be readily applied to a wide range of related numerical methods. However, the impact of the implementation on numerical properties such as dispersion, diffusion and accuracy is expected to differ.

The approach is based on solving the inverse Riemann problem and differs substantially from the typically used traction-at-split-node (TSN) technique (Andrews, 1999; Day et al., 2005; Dalguer and Day, 2007). A Riemann problem is defined as an initial-value problem for a conservation law (here the hyperbolic partial differential equation) in which initial conditions contain a spatial discontinuity. Naturally, this problem occurs in finite volume (FV) and DG methods across each element face. We solve it using the exact Riemann solver, also known as Godunov flux, which is a wellknown concept in numerical analysis. This provides a smooth solution along the element face, the Godunov state, despite the element-wise discontinuous approximation of the physical unknowns. The most important benefit of this flux-based method compared to existing methods is the absence of spurious modes in the slip rate.

We consider a fault in the $x=0$ plane. An arbitrary fault side is defined as the + side, the opposing one as the - side. $\sigma_{i j}$ denotes the relative stress tensor (stress changes relative to the initial stresses), $u, v$ and $w$ the particle velocities in the $x, y$ and $z$ directions, respectively, $\Delta v=v^{+}-v^{-}$and $\Delta w=w^{+}-w^{-}$the two components of slip rate, $\rho$ density, $c_{\mathrm{P}} \mathrm{P}$ wave velocity and $c_{\mathrm{S}} \mathrm{S}$ wave velocity. Variables denoted with superscript 0 are the corresponding initial values and those denoted with a superscript $G$ correspond to the Godunov state.

We enforce the Coulomb failure criterion at each integration point of the DG method located on the fault surface:

$|\tau| \leq \tau_{\mathrm{S}}$,

where $|\tau|=\sqrt{\left(\sigma_{x y}+\sigma_{x y}^{0}\right)^{2}+\left(\sigma_{x z}+\sigma_{x z}^{0}\right)^{2}}$ is the absolute shear traction and $\tau_{\mathrm{S}}=\mu_{\mathrm{f}}\left(\sigma_{x x}+\sigma_{x x}^{0}\right)$ is the fault strength. The friction coefficient $\mu_{\mathrm{f}}$ may be a function of slip, slip rate, state evolution variables and other frictional parameters.

We compute the Godunov state accounting for a material contrast across the fault, based on Toro (1999), LeVeque
(2002), de la Puente et al. (2009), Pelties et al. (2012), as follows:

$$
\begin{aligned}
\sigma_{x x}^{\mathrm{G}} & =\sigma_{x x}^{+}+\frac{\left[\left(\sigma_{x x}^{-}-\sigma_{x x}^{+}\right)+c_{\mathrm{p}}^{-} \rho^{-}\left(u^{-}-u^{+}\right)\right] c_{\mathrm{p}}^{+} \rho^{+}}{c_{\mathrm{p}}^{+} \rho^{+}+c_{\mathrm{p}}^{-} \rho^{-}}, \\
\sigma_{x y}^{\mathrm{G}} & =\sigma_{x y}^{+}+\frac{\left[\left(\sigma_{x y}^{-}-\sigma_{x y}^{+}\right)+c_{\mathrm{s}}^{-} \rho^{-}\left(v^{-}-v^{+}\right)\right] c_{\mathrm{s}}^{+} \rho^{+}}{c_{\mathrm{s}}^{+} \rho^{+}+c_{\mathrm{s}}^{-} \rho^{-}}, \\
\sigma_{x z}^{\mathrm{G}} & =\sigma_{x z}^{+}+\frac{\left[\left(\sigma_{x z}^{-}-\sigma_{x z}^{+}\right)+c_{\mathrm{s}}^{-} \rho^{-}\left(w^{-}-w^{+}\right)\right] c_{\mathrm{s}}^{+} \rho^{+}}{c_{\mathrm{s}}^{+} \rho^{+}+c_{\mathrm{s}}^{-} \rho^{-}}, \\
u^{\mathrm{G}} & =u^{+}+\frac{\sigma_{x x}^{\mathrm{G}}-\sigma_{x x}^{+}}{c_{\mathrm{p}}^{+} \rho^{+}}, \\
v^{\mathrm{G}} & =v^{+}+\frac{\sigma_{x y}^{\mathrm{G}}-\sigma_{x y}^{+}}{c_{\mathrm{s}}^{+} \rho^{+}}, \\
w^{\mathrm{G}} & =w^{+}+\frac{\sigma_{x z}^{\mathrm{G}}-\sigma_{x z}^{+}}{c_{\mathrm{s}}^{+} \rho^{+}} .
\end{aligned}
$$

Quantities associated to zero wave speeds, namely $\sigma_{y y}, \sigma_{z z}$ and $\sigma_{y z}$, do not contribute to the solution of the Riemann problem and are ignored.

The Godunov state is the solution of the elastic wave equation in the absence of a fault. When a fault is present, it can be viewed as the state predicted in the absence of (further) slip, and the Godunov fault tractions play the same role as the "stick tractions" in the split-node method (Kaneko et al., 2008). If the absolute shear traction in the Godunov state satisfies $\left|\tau^{\mathrm{G}}\right| \leq \tau_{\mathrm{S}}$, the fault point is locked and the stresses and velocities are assigned to the values given by the Godunov state. If $\left|\tau^{\mathrm{G}}\right|>\tau_{\mathrm{S}}$, the fault point fails, slip is declared, and we impose the relative shear stresses to be

$$
\begin{gathered}
\tilde{\sigma}_{x y}=\frac{\sigma_{x y}^{\mathrm{G}}+\sigma_{x y}^{0}}{\left|\tau^{\mathrm{G}}\right|} \tau_{\mathrm{S}}-\sigma_{x y}^{0}, \\
\tilde{\sigma}_{x z}=\frac{\sigma_{x z}^{\mathrm{G}}+\sigma_{x z}^{0}}{\left|\tau^{\mathrm{G}}\right|} \tau_{\mathrm{S}}-\sigma_{x z}^{0} .
\end{gathered}
$$

and - sides as

$$
\begin{gathered}
\tilde{v}^{+}=v^{+}+\frac{\tilde{\sigma}_{x y}-\sigma_{x y}^{+}}{c_{\mathrm{s}}^{+} \rho^{+}}, \\
\tilde{v}^{-}=v^{-}-\frac{\tilde{\sigma}_{x y}-\sigma_{x y}^{-}}{c_{\mathrm{s}}^{-} \rho^{-}}, \\
\tilde{w}^{+}=w^{+}+\frac{\tilde{\sigma}_{x z}-\sigma_{x z}^{+}}{c_{\mathrm{s}}^{+} \rho^{+}}, \\
\tilde{w}^{-}=w^{-}-\frac{\tilde{\sigma}_{x z}-\sigma_{x z}^{-}}{c_{\mathrm{s}}^{-} \rho^{-}} .
\end{gathered}
$$

Godunov variables $v^{\mathrm{G}}, w^{\mathrm{G}}, \sigma_{x y}^{\mathrm{G}}$ and $\sigma_{x z}^{\mathrm{G}}$ given in Eq. (A2) and subtracting the corresponding equations in Eq. (A4):

$$
\begin{aligned}
\Delta \tilde{v} & =\left(\frac{1}{c_{\mathrm{s}}^{+} \rho^{+}}+\frac{1}{c_{\mathrm{s}}^{-} \rho^{-}}\right)\left(\tilde{\sigma}_{x y}-\sigma_{x y}^{\mathrm{G}},\right. \\
\Delta \tilde{w} & =\left(\frac{1}{c_{\mathrm{s}}^{+} \rho^{+}}+\frac{1}{c_{\mathrm{s}}^{-} \rho^{-}}\right)\left(\tilde{\sigma}_{x z}-\sigma_{x z}^{\mathrm{G}}\right) .
\end{aligned}
$$



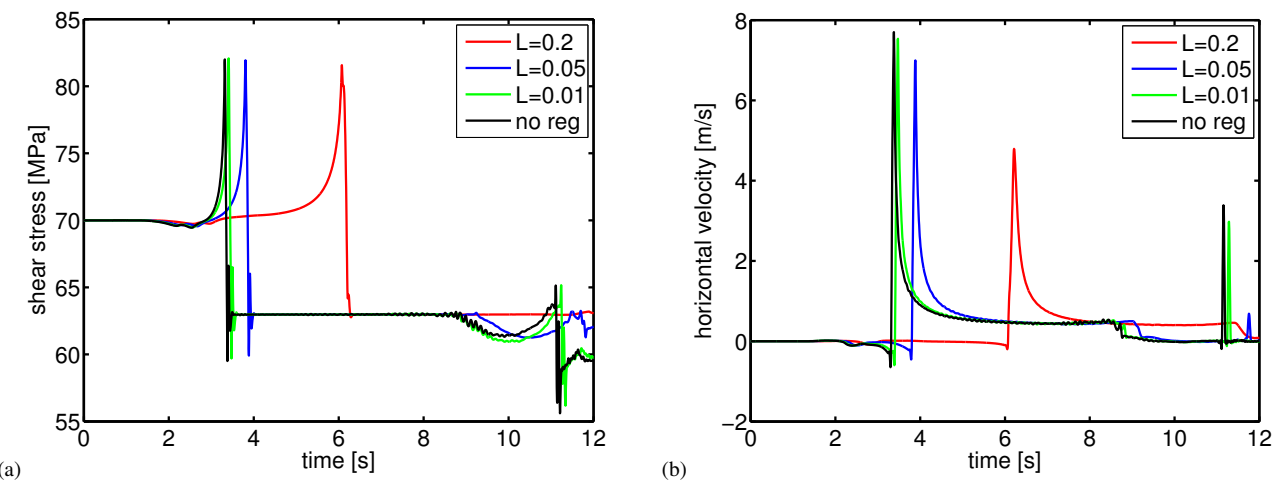

Fig. B1. Influence of Prakash-Clifton regularization on the ADER-DG solution of the ill-posed bimaterial problem TPV7. Time series of stress and velocity at a surface location on the far side of the fault above the nucleation zone for different values of the characteristic regularization length-scale $L$ indicated in the legend (in meters).

In summary, this implementation allows modeling fault dynamics in bimaterial faults and features the same numerical properties as in the homogeneous case. For identical material properties at the + and - sides of the fault the above equations yield the equations presented in Pelties et al. (2012).

\section{Appendix B}

\section{Influence of Prakash-Clifton regularization}

As noted in the introduction of Sect. 6, ill-posed bimaterial problems need to be regularized to ensure convergence. Regularization can be achieved by Prakash-Clifton regularization as introduced in Prakash and Clifton (1993) and Cochard and Rice (2000), which is a laboratory-based law relating the shear strength evolution continuously to abrupt changes of normal stress. We implement optional Prakash-Clifton regularization (not used for the benchmark presented in Sect. 6) in the simplified form

$\dot{\tau}^{\mathrm{S}}=-\frac{|V|+V^{*}}{L}\left(\tau^{\mathrm{S}}-\mu \max \left(0,-\sigma_{n}\right)\right)$,

where $\tau^{\mathrm{S}}$ is the frictional strength, $V$ is the slip velocity, $V^{*}$ the characteristic slip velocity, $L$ a characteristic distance, $\mu$ the friction coefficient, and $\sigma_{n}$ the normal stress. Note that the characteristic distance introduced here is not related to $L$ in Sect. 7.

Evidently, the amount of added damping or regularization could have a significant influence on the result as elaborated by Cochard and Rice (2000). Therefore, we present here the influence of the regularization on the ADER-DG algorithm to provide guidance for users on the impact of regularization. To this end, we summarize in the following our findings from the ill-posed SCEC test case scenario TPV7. The setup is identical to TPV6 except for the far side featuring a wave speed reduction of $20 \%$ in combination with equal material densities at both sides of the fault. We apply a resolution of $h=200 \mathrm{~m}$ and $\mathcal{O} 4$ in space and time.

We set $V^{*}$ to $1 \mathrm{~m} \mathrm{~s}^{-1}$ and vary the characteristic distance $L$. Figure B1 compares results with $L$ of $0.2,0.05$, and $0.01 \mathrm{~m}$ and without regularization. As expected, the $L$ values of 0.2 and $0.05 \mathrm{~m}$ lead to large discrepancies in peak slip rate and rupture time. However, even a small characteristic distance $L$ of $0.01 \mathrm{~m}$ produces visible differences in shear stress and slip rate.

As we reduce $L$ we find convergence towards the solution without Prakash-Clifton regularization, instead of the noisy solution expected for the ill-posed problem solved with a high resolution (small element edge length $h$ and high order of accuracy $\mathcal{O}$ ) (Cochard and Rice, 2000). We conclude that there is an additional source of regularization in our simulations: the intrinsic numerical dissipation of ADERDG with Godunov fluxes (see Sect. 2). This is not necessarily trivial: other forms of numerical dissipation may not be strong enough to counteract the growth of the unstable shortwavelength modes, as needed to regularize the problem. Our simulations then contain two efficient regularization mechanisms: (1) Prakash-Clifton regularization and (2) ADERDG's numerical dissipation. The characteristic timescale of the Prakash-Clifton regularization is $L / V^{*}$. The cutoff period of ADER-DG's dissipation is $\sim h / \mathcal{O} / c_{\mathrm{S}}$. Only in the simulation with $L$ of $0.01 \mathrm{~m}$ is the former shorter than the latter. This explains why the effect of the Prakash-Clifton regularization is much less apparent if $L$ is $0.01 \mathrm{~m}$. We note that, because ADER-DG's intrinsic numerical dissipation depends on resolution ( $h$ and $\mathcal{O}$ ), the frequencies that are well regularized for a given resolution can be more unstable at a finer resolution. Our analysis of regularization timescales provides a criterion to set regularization and resolution parameters in ADER-DG simulations of bimaterial rupture in the ill-posed regime. 
Acknowledgements. We thank the Southern California Earthquake Center, especially Ruth Harris and Michael Barall, for hosting the Spontaneous Rupture Code Verification Project, Yoshihiro Kaneko for helpful discussions on the implementation of rate-and-state friction and Amaryllis Nerger for her preparatory work on the 2-D version of the branching fault benchmark. Christian Pelties was funded through the Emmy Noether Programme (KA 2281/2-1) of the Deutsche Forschungsgemeinschaft and by the Volkswagen Stiftung (ASCETE project). Alice-Agnes Gabriel was funded by the Deutsche Forschungsgemeinschaft (KA 4-1) and Jean-Paul Ampuero by the US NSF (CAREER award EAR-1151926) and SCEC (based on NSF Cooperative Agreement EAR-0529922 and USGS Cooperative Agreement 07HQAC0026). BaCaTeC supported research visits for Jean-Paul Ampuero and Christian Pelties at LMU and Caltech, respectively. The computations were performed on SuperMUC at LRZ, Garching, Germany, and Shaheen at KAUST, Saudi Arabia.

Edited by: L. Gross

\section{References}

Abrahamson, N. A. and Somerville, P. G.: Effects of the hanging wall and footwall on ground motions recorded during the Northridge earthquake, Bull. Seism. Soc. Am., 86, S93-S99, 1996.

Adams, G. G.: Self-excited oscillations of two elastic half-spaces sliding with a constant coefficient of friction, J. App. Mech., 62, 867-872, 1995.

Ampuero, J.-P.: SEM2DPACK: A spectral element method for 2D wave propagation and earthquake source dynamics, version 2.3.3, available at: http://sourceforge.net/projects/sem2d/, 2008.

Ampuero, J.-P. and Ben-Zion, Y.: Cracks, pulses and macroscopic asymmetry of dynamic rupture on a bimaterial interface with velocity-weakening friction, Geophys. J. Int., 173, 674-692, doi:10.1111/j.1365-246X.2008.03736.x, 2008.

Ampuero, J.-P., Ripperger, J., and Mai, P. M.: Properties of dynamic earthquake ruptures with heterogeneous stress drop, in: Earthquakes: radiated energy and the physics of faulting, edited by: Abercrombie, R., McGarr, A., Di Toro, G., and Kanamori, H., 255-261, Wiley Online Library, doi:10.1029/170GM25, 2006.

Andrews, D. J.: Test of two methods for faulting in finite-difference calculations, Bull. Seism. Soc. Am., 89, 931-937, 1999.

Andrews, D. J.: Rupture dynamics with energy loss outside the slip zone, J. Geophys. Res., 110, B01307, doi:10.1029/2004JB003191, 2005.

Andrews, D. J., Hanks, T. C., and Whitney, J. W.: Physical limits on ground motion at Yucca Mountain, Bull. Seism. Soc. Am., 97, 1771-1792, 2007.

Aochi, H., Madariaga, R., and Fukuyama, E.: Constraint of fault parameters inferred from nonplanar fault modeling, Geochem. Geophys. Geosys., 4, 1020, doi:10.1029/2001GC000207, 2003.

Barall, M.: A grid-doubling finite-element technique for calculating dynamic three-dimensional spontaneous rupture on an earthquake fault, Geophys. J. Int., 178, 845-859, doi:10.1111/j.1365246X.2009.04190.x, 2009.

Barbot, S., Lapusta, N., and Avouac, J.-P.: Under the hood of the earthquake machine: toward predictive modeling of the seis- mic cycle, Science, 336, 707-710, doi:10.1126/science.1218796, 2012.

Bhat, H. S., Olives, M., Dmowska, R., and Rice, J. R.: Role of fault branches in earthquake rupture dynamics, J. Geophys. Res., 112, 2156-2202, doi:10.1029/2007JB005027, 2007.

Boatwright, J. and Quin, H.: The seismic radiation from a 3-D dynamic model of a complex rupture process; Part I, Confined ruptures, in: Earthquake source mechanics, edited by: Das, S., Boatwright, J., and Scholz, C. H., Geophysical Monograph, Am. Geophys. Union, 37, 97-109, 1986.

Brace, W. F. and Byerlee, Z. D.: Stick-slip as a mechanism for earthquakes, Science, 153, 990-992, doi:10.1126/science.153.3739.990, 1966.

Brietzke, G. B., Cochard, A., and Igel, H.: Importance of bimaterial interfaces for earthquake dynamics and strong ground motion, Geophys. J. Int., 178, 921-938, doi:10.1111/j.1365246X.2009.04209.x, 2009.

Cochard, A. and Madariaga, R.: Dynamic faulting under ratedependent friction, Pure Appl. Geophys., 142, 419-445, 1994.

Cochard, A. and Rice, J. R.: Fault rupture between dissimilar materials: ill-posedness, regularization, and slippulse response, J. Geophys. Res., 105, 25891-25907, doi:10.1029/2000JB900230, 2000.

Cui, Y., Olsen, K. B., Jordan, T. H., Lee, K., Zhou, J., Small, P., Roten, D., Ely, G., Panda, D. K., Chourasia, A., Levesque, J., Day, S. M., and Maechling, P.: Scalable earthquake simulation on petascale supercomputers, in: International Conference for High Performance Computing, Networking, Storage and Analysis (SC), 1-20, doi:10.1109/SC.2010.45, 2010.

Dalguer, L. A. and Day, S. M.: Staggered-grid split-node method for spontaneous rupture simulation, J. Geophys. Res., 112, B02302, doi:10.1029/2006JB004467, 2007.

Dalguer, L. A., Irikura, K., Riera, J. D., and Chiu, H. C.: The importance of the dynamic source effects on strong ground motion during the 1999 Chi-Chi, Taiwan, earthquake: Brief interpretation of the damage distribution on buildings, Bull. Seism. Soc. Am., 91, 1112-1127, 2001.

Daub, E. G., Manning, M. L., and Carlson, J. M.: Pulselike, crack-like, and supershear earthquake ruptures with shear strain localization, J. Geophys. Res., 115, B05311, doi:10.1029/2009JB006388, 2010.

Day, S. M.: Three-dimensional finite-difference simulation of fault dynamics: rectangular faults with fixed rupture velocity, Bull. Seism. Soc. Am., 72, 705-727, 1982.

Day, S. M., Yu, G., and Wald, D. J.: Dynamic stress changes during earthquake rupture, Bull. Seism. Soc. Am., 88, 512-522, 1998.

Day, S. M., Dalguer, L. A., Lapusta, N., and Liu, Y.: Comparison of finite difference and boundary integral solutions to three-dimensional spontaneous rupture, J. Geophys. Res., 110, B12307, doi:10.1029/2005JB003813, 2005.

DeDontney, N., Rice, J. R., and Dmowska, R.: Influence of material contrast on fault branching behavior, Geophys. Res. Lett., 38, L14305, doi:10.1029/2011GL047849, 2011.

de la Puente, J., Ampuero, J.-P., and Käser, M.: Dynamic rupture modeling on unstructured meshes using a discontinuous Galerkin method, J. Geophys. Res., 114, B10302, doi:10.1029/2008JB006271, 2009. 
Di Toro, G., Nielsen, S. B., and Pennacchioni, G.: Earthquake rupture dynamics frozen in exhumed ancient faults, Nature, 436, 1009-1012, doi:10.1038/nature03910, 2005.

Di Toro, G., Han, R., Hirose, T., De Paola, N., Nielsen, S. B., Mizoguchi, K., Ferri, F., Cocco, M., and Shimamoto, T.: Fault lubrication during earthquakes, Nature, 471, 494-498, doi:10.1038/nature09838, 2011.

Dieterich, J. H.: Time-dependent friction and the mechanics of stick-slip, Pure Appl. Geophys., 116, 790-806, doi:10.1007/BF00876539, 1978.

Duan, B. and Day, S. M.: Inelastic strain distribution and seismic radiation from rupture of a fault kink, J. Geophys. Res., 113, 21562202, doi:10.1029/2008JB005847, 2008.

Dumbser, M. and Käser, M.: An arbitrary high order discontinuous Galerkin method for elastic waves on unstructured meshes II: The three-dimensional case, Geophys. J. Int., 167, 319-336, 2006.

Dunham, E. M.: Dissipative interface waves and the transient response of a three-dimensional sliding interface with Coulomb friction, J. Mech. Phys. Sol., 53, 327-357, doi:10.1016/j.jmps.2004.07.003, 2005.

Dunham, E. M.: Conditions governing the occurrence of supershear ruptures under slip-weakening friction, J. Geophys. Res., 112, 1-24, 2007.

Dunham, E. M.: MDSBI: Multi-dimensional spectral boundary integral code, version 4.1.7, available at: http://pangea.stanford. edu/ edunham/codes/codes.html, 2008.

Dunham, E. M., Belanger, D., Cong, L., and Kozdon, J. E.: Earthquake ruptures with strongly rate-weakening friction and offfault plasticity, part 1: planar faults, Bull. Seism. Soc. Am., 101, 2296-2307, 2011.

Ely, G. P., Day, S. M., and Minster, J.-B.: Dynamic rupture models for the Southern San Andreas fault, Bull. Seis. Soc. Am., 100, 131-150, doi:10.1785/0120090187, 2010.

Gabriel, A.-A., Ampuero, J.-P., Dalguer, L. A., and Mai, P. M.: The transition of dynamic rupture modes in elastic media under velocity-weakening friction, J. Geophys. Res., 117, 0148-0227, doi:10.1029/2012JB009468, 2012.

Gabriel, A.-A., Ampuero, J.-P., Dalguer, L. A., and Mai, P. M.: Source properties of dynamic rupture pulses with off-fault plasticity, J. Geophys. Res. Solid Earth, 118, 4117-4126, doi:10.1002/jgrb.50213, 2013.

Harris, R. A. and Day, S. M.: Effects of a low-velocity zone on a dynamic rupture, Bull. Seism. Soc. Am., 87, 1267-1280, 1997.

Harris, R. A., Barall, M., Archuleta, R., Dunham, E., Aagaard, B., Ampuero, J.-P., Bhat, H., Cruz-Atienza, V., Dalguer, L. A., Dawson, P., Day, S. M., Duan, B., Ely, G., Kaneko, Y., Kase, Y., Lapusta, N., Liu, Y., Ma, S., Oglesby, D., Olsen, K., Pitarka, A., Song, S., and Templeton, E. L.: The SCEC/USGS dynamic earthquake rupture code verification exercise, Seismol. Res. Lett., 80, 119-126, doi:10.1785/gssrl.80.1.119, 2009.

Harris, R. A., Barall, M., Andrews, D. J., Duan, B., Ma, S., Dunham, E. M., Gabriel, A.-A., Kaneko, Y., Kase, Y., Aagaard, B. T., Oglesby, D. D., Ampuero, J.-P., Hanks, T. C., and Abrahamson, N.: Verifying a computational method for predicting extreme ground motion, Seismol. Res. Lett., 82, 638-644, doi:10.1785/gssrl.82.5.638, 2011.
Huang, Y. and Ampuero, J.-P.: Pulse-like ruptures induced by low-velocity fault zones, J. Geophys. Res., 116, B12307, doi:10.1029/2011JB008684, 2011.

Huang, Y., Ampuero, J.-P., and Kanamori, H.: Slip-weakening models of the 2011 Tohoku-Oki earthquake and constraints on stress drop and fracture energy, Pure Appl. Geophys., 1-14, doi:10.1007/s0002401307182, 2013.

Hughes, T. J. R.: The finite element method: linear static and dynamic finite element analysis, Dover Civil and Mechanical Engineering Series, Dover Publications, 2000.

Kaneko, Y., Lapusta, N., and Ampuero, J.-P.: Spectral element modeling of spontaneous earthquake rupture on rate and state faults: effect of velocity-strengthening friction at shallow depths, J. Geophys. Res., 113, B09317, doi:10.1029/2007JB005553, 2008.

Karypis, G. and Kumar, V.: Multilevel k-way partitioning scheme for irregular graphs, J. Parallel Distrib. Comput., 48, 96-129, 1998.

Käser, M. and Dumbser, M.: An arbitrary high order discontinuous Galerkin method for elastic waves on unstructured meshes I: The two-dimensional isotropic case with external source terms, Geophys. J. Int., 166, 855-877, 2006.

Käser, M., Hermann, V., and de la Puente, J.: Quantitative accuracy analysis of the discontinuous Galerkin method for seismic wave propagation, Geophys. J. Int., 173, 990-999, doi:10.1029/2008JB005934, 2008.

Lapusta, N. and Liu, Y.: Three-dimensional boundary integral modeling of spontaneous earthquake sequences and aseismic slip, J. Geophys. Res., 114, B09303, doi:10.1029/2008JB005934, 2009.

LeVeque, R. L.: Finite volume methods for hyperbolic problems, Cambridge University Press, Cambridge, 2002.

Ma, S. and Andrews, D. J.: Inelastic off-fault response and three-dimensional dynamics of earthquake rupture on a strike-slip fault, J. Geophys. Res., 115, B04304, doi:10.1029/2009JB006382, 2010.

Ma, S. and Archuleta, R. J.: Radiated seismic energy based on dynamic rupture models of faulting, J. Geophys. Res., 111, B05315, doi:10.1029/2005JB004055, 2006.

Ma, S. and Beroza, G. C.: Rupture dynamics on a bimaterial interface for dipping faults, Bull. Seism. Soc. Am., 98, 1642-1658, doi:10.1785/0120070201, 2008.

Madariaga, R.: Radiation from a finite reverse fault in a half space, Pure Appl. Geophys., 160, 555-577, doi:10.1007/PL00012550, 2003.

Niemeijer, A., Marone, C., and Elsworth, D.: Frictional strength and strain weakening in simulated fault gouge: Competition between geometrical weakening and chemical strengthening, J. Geophys. Res., 115, B10207, doi:10.1029/2009JB000838, 2010.

Noda, H., Dunham, E. M., and Rice, J. R.: Earthquake ruptures with thermal weakening and the operation of major faults at low overall stress levels, J. Geophys. Res., 114, B07302, doi:10.1029/2008JB006143, 2009.

Oglesby, D. D. and Day, S. M.: Stochastic fault stress: implications for fault dynamics and ground motion, Bull. Seism. Soc. Am., 92, 3006-3021, 2002.

Oglesby, D. D., Archuleta, R. J., and Nielsen, S. B.: Earthquakes on dipping faults: the effects of broken symmetry, Science, 280, 1055-1059, doi:10.1126/science.280.5366.1055, 1998. 
Oglesby, D. D., Day, S. M., Li, Y.-G., and Vidale, J. E.: The 1999 Hector Mine earthquake: the dynamics of a branched fault system, Bull. Seism. Soc. Am., 93, 2459-2476, doi:10.1785/0120030026, 2003.

Ohnaka, M. and Kuwahara, Y.: Characteristic features of local breakdown near a crack-tip in the transition zone from nucleation to unstable rupture during stick-slip shear failure, Tectonophysics, Earthquake Source Processes, 175, 197-220, 1990.

Pacheco, J. F. and Sykes, L. R.: Seismic moment catalog of large shallow earthquakes, 1900 to 1989, Bull. Seism. Soc. Am., 82, 1306-1349, 1992.

Pelties, C., Käser, M., Hermann, V., and Castro, C.: Regular vs. irregular meshing for complicated models and their effect on synthetic seismograms, Geophys. J. Int., 183, 1031-1051, doi:10.1111/j.1365-246X.2010.04777.x, 2010.

Pelties, C., de la Puente, P., Ampuero, J.-P., Brietzke, G. B., and Käser, M.: Three-dimensional dynamic rupture simulation with a high-order discontinuous Galerkin method on unstructured tetrahedral meshes, J. Geophys. Res., 117, B02309, doi:10.1029/2011JB008857, 2012.

Prakash, V. and Clifton, R. J.: Time resolved dynamic friction measurements in pressure shear, Experimental Techniques in the Dynamics of Deformable Solids, 165, 33-48, 1993.

Rice, J. R.: Heating and weakening of faults during earthquake slip, J. Geophys. Res., 111, B05311, doi:10.1029/2005JB004006, 2006.

Rice, J. R., Lapusta, N., and Ranjith, K.: Rate and state dependent friction and the stability of sliding between elastically deformable solids, J. Mech. Phys. Solids, 49, 1865-1898, 2001.

Rojas, O., Day, S. M., Castillo, J., and Dalguer, L. A.: Modelling of rupture propagation using high-order mimetic finite differences, Geophys. J. Int., 172, 631-650, doi:10.1111/j.1365246X.2007.03651.x, 2008.
Roten, D., Olsen, K. B., Pechmann, J. C., Cruz-Atienza, V. M., and Magistrale, H.: 3D simulations of M7 earthquakes on the Wasatch fault, Utah, part I: long-period $(0-1 \mathrm{~Hz})$ ground motion, Bull. Seis. Soc. Am., 101, 2045-2063, doi:10.1785/0120110031, 2011.

Rudnicki, J. W. and Wu, M.: Mechanics of dip-slip faulting in an elastic half-space, J. Geophys. Res., 100, 22122-22173, 1995.

Ruina, A.: Slip Instability and State Variable Friction Laws, J. Geophys. Res., 88, 10359-10370, doi:10.1029/JB088iB12p10359, 1983.

Schwartz, D. P., Haeussler, P. J., Seitz, G. G., and Dawson, T. E.: Why the 2002 Denali fault rupture propagated onto the Totschunda fault: Implications for fault branching and seismic hazards, J. Geophys. Res., 117, B11304, doi:10.1029/2011JB008918, 2012.

Templeton, E. L. and Rice, J. R.: Off-fault plasticity and earthquake rupture dynamics: 1. Dry materials or neglect of fluid pressure changes, J. Geophys. Res., 113, B09306, doi:10.1785/0120050825, 2008.

Thurber, C., Zhang, H., Waldhauser, F., Hardebeck, J., Michael, A., and Eberhart-Phillips, D.: Three-dimensional compressional wavespeed model, earthquake relocations, and focal mechanisms for the Parkfield, California, region, Bull. Seism. Soc. Am., 96, S38-S49, 2006.

Toro, E. F.: Riemann solvers and numerical methods for fluid dynamics, Springer, 2nd Edn., 1999.

Zhou, J., Cui, Y., Poyraz, E., Choi, D. J., and Guest, C. C.: MultiGPU Implementation of a 3D Finite Difference Time Domain Earthquake Code on Heterogeneous Supercomputers, in: Proceedings of the International Conference on Computational Science (ICCS), Barcelona, Spain, 5-7 June, 2013, 1255-1264, doi:10.1016/j.procs.2013.05.292, 2013. 